Why do human languages have homophones?

2

Sean Trott and Benjamin Bergen

4 Department of Cognitive Science, UC San Diego, 9500 Gilman Dr., La Jolla, CA 92093

5

Sean Trott (Corresponding author):

Email address: sttrott@ucsd.edu

Mailing address:

Department of Cognitive Science

10 9500 Gilman Dr.

11

La Jolla, CA 92093-0515

12 


\section{WHY DO HUMAN LANGUAGES HAVE HOMOPHONES?}

\section{Abstract}

Human languages are replete with ambiguity. This is most evident in homophony-where two or more words sound the same, but carry distinct meanings. For example, the wordform "bark" can denote either the sound produced by a dog or the protective outer sheath of a tree trunk. Why would a system evolved for efficient, effective communication display rampant ambiguity? Some accounts argue that ambiguity is actually a design feature of human communication systems, allowing languages to recycle their most optimal wordforms (those which are short, frequent, and phonotactically well-formed) for multiple meanings. We test this claim by constructing five series of artificial lexica matched for the phonotactics and distribution of word lengths found in five real languages (English, German, Dutch, French, and Japanese), and comparing both the quantity and concentration of homophony across the real and artificial lexica. Surprisingly, we find that the artificial lexica exhibit higher upper-bounds on homophony than their real counterparts, and that homophony is even more likely to be found among short, phonotactically plausible wordforms in the artificial than in the real lexica. These results suggest that homophony in real languages is not directly selected for, but rather, that it emerges as a natural consequence of other features of a language. In fact, homophony may even be selected against in real languages, producing lexica that better conform to other requirements of humans who need to use them. Finally, we explore the hypothesis that this is achieved by "smoothing" out dense concentrations of homophones across lexical neighborhoods, resulting in comparatively more minimal pairs in real lexica.

Keywords: homophones; ambiguity; efficiency; language evolution; phonotactics 


\section{WHY DO HUMAN LANGUAGES HAVE HOMOPHONES?}

\section{Introduction}

38 Human languages are replete with ambiguity. This is most evident in homophony — where two or

39 more words sound the same, but carry distinct meanings. For example, the wordform "bark" can

40 denote either the sound produced by a dog or the protective outer sheath of a tree trunk.

41 Estimates of the rate of homophony in English range from 7.4\% (Rodd et al, 2002) to over $15 \%{ }^{1}$

42 (Baayen et al, 1995). Dautriche (2015) estimates the average homophony rate across languages

43 to be $4 \%$, with considerable cross-linguistic variability, ranging from approximately $3 \%$ in Dutch

44 to $15 \%$ in Japanese. The prevalence of homophony, like other kinds of ambiguity, is

45 confounding on its face. Human languages are generally thought to be shaped by pressures for

46 efficient, effective communication (Zipf, 1949; Gibson et al, 2019). Yet ambiguity increases both

47 the effort required for comprehension and the likelihood of miscommunication. A comparison

48 between human and programming languages places this into relief. Programming languages,

49 designed for efficient and errorless communication, generally abide no ambiguity at all. Why

50 then do human languages insist on encoding distinct messages identically? Why are homophones

51 so common?

52 Part of the answer appears to be that human comprehenders are adept at disambiguating

53 ambiguous input using various contextual cues (Levinson, 2000; Wasow et al, 2005; Ferreira,

54 2008; Piantadosi et al, 2012). In the case of homophones, a wide array of cues to meaning are

55 available, including the syntactic structures that words are embedded in (Dautriche et al, 2018),

56 gestures that accompany speech (Holle \& Gunter, 2007; Holler \& Beattie, 2003; Kidd \& Holler,

57 2009), and statistical aspects of linguistic context (Aina et al, 2019). The human capacity for

\footnotetext{
${ }^{1}$ Estimates of the rate of polysemy (wordforms with related meanings) are considerably higher: up to $80 \%$ of wordforms in English are thought to be polysemous (Rodd et al, 2002).
} 


\section{WHY DO HUMAN LANGUAGES HAVE HOMOPHONES?}

58 disambiguation thus creates a tolerant environment for ambiguous wordforms — explaining why

59 as languages evolve, homophones might not be strictly selected against.

But might homophones also be selected for? Zipf (1949) argues that ambiguity is a

61 design feature of any human communication system, resulting from a direct pressure for

62 efficiency. A growing body of evidence is consistent with the claim that lexica are optimized for

63 efficient communication between humans (Piantadosi et al, 2009; Gibson et al, 2019), from the

64 way they carve up semantic domains (Regier et al, 2007; Kemp \& Regier, 2012; Xu \& Regier,

65 2014; Kemp et al, 2018; Zaslavsky et al, 2018) to the wordforms that they contain (Piantadosi et

66 al, 2011; Piantadosi et al, 2012; Mahowald et al, 2018). This pressure for an efficient lexicon

67 could result in a selective bias for wordforms that are particularly easy to produce and

68 comprehend, where ease reflects properties such as a word's length, phonotactic plausibility, and

69 frequency. Combined with a tolerance for ambiguity, a bias for easy wordforms could exert a

70 pressure on lexica to "recycle" particularly optimal wordforms for multiple meanings. This

71 pressure, termed "unification" by Zipf (1949), would increase efficiency by reducing the number

72 of unique wordforms that speakers need to learn and encode. Furthermore, by preferentially re-

73 using the most optimal wordforms, such a lexicon would arguably involve less effort in speaking

74 or writing than an unambiguous linguistic system. If such a pressure exists, it should produce

75 concentrations of homophony in optimal regions of phonotactic space - the "easiest" wordforms

76 should be the most ambiguous. Indeed, Piantadosi et al (2012) find that English, German, and

77 Dutch count more homophones among wordforms that are short, frequent, and phonotactically

78 well-formed. This finding is consistent with the idea that ambiguity arises out of a pressure for

79 efficiency. 


\section{WHY DO HUMAN LANGUAGES HAVE HOMOPHONES?}

However, homophony could also emerge in a lexicon without being directly selected for, as an indirect consequence of other factors affecting how words are distributed in a lexicon. Two

82 indirect mechanisms could also partially (or even fully) account for the uneven distribution of

83 homophony across a lexicon.

First, the proportion of occupied phonotactic space (i.e., the ratio of actual wordforms to

85 possible wordforms) for English and every other language we are aware of will always be higher

86 for shorter wordforms than for longer wordforms. This is because the number of possible

87 wordforms of a given length grows exponentially with each added syllable. If a language's

88 phonotactics permit $n$ unique syllables, then there are $n$ possible monosyllabic wordforms,

89 approximately $n^{2}$ possible bisyllabic wordforms, approximately $n^{3}$ possible trisyllabic

90 wordforms, and so on. In contrast, the number of actual wordforms does not grow exponentially

91 with word length (e.g., the CELEX set of English lemmas contains approximately 7706

92 monosyllabic words, 15247 disyllabic words, and 11379 trisyllabic words). This means that the

93 proportion of occupied phonotactic space will always be greater among short wordforms than

94 long wordforms. Thus, even if words were randomly added to a lexicon, homophony would by

95 chance be more likely to occur among short wordforms than long wordforms.

Second, the existence of phonotactic constraints results in a lexicon that is not uniformly

97 distributed across the space of possible wordforms. All languages appear to impose idiosyncratic

98 constraints on sounds and their combinations - for example, English does not allow the velar

99 nasal $/ y /$ in syllable onsets, unlike Vietnamese; but English does allow consonant clusters like

100 /st/, unlike Japanese. Phonotactic regularities narrow the space of possible wordforms

101 considerably (Dautriche et al, 2017). By limiting the range of possible wordforms and biasing the

102 formation and evolution of the lexicon, these phonotactic constraints could also increase the 


\section{WHY DO HUMAN LANGUAGES HAVE HOMOPHONES?}

103 prevalence of homophones. Critically, they could do so even without a direct pressure to reuse

104 entire wordforms. Even a pressure to merely statistically reuse certain phonological sequences

105 more often would increase the likelihood of homophones overall, and particularly among the

106 most phonotactically probable wordforms.

107 Both of these mechanisms offer indirect causal pathways whereby a drive for efficiency

108 could lead to increased homophony. For example, more phonotactically regular words could be

109 easier to learn (Jusczyk et al, 1994; Gathercole et al, 1991; Munson, 2001; Coady \& Aslin,

110 2004), which would lead to more phonotactically probable words being more likely to be

111 transmitted across generations, or less phonotactically words becoming more phonotactically

112 probable through imperfect intergenerational transmission. This in turn could result in increased

113 homophony, particularly among highly probable wordforms. Once again, though, both

114 phonotactics and the distribution of word lengths in a lexicon could in principle lead to the

115 emergence of homophony without a direct, selective pressure for the preferential reuse of

116 specific, optimal wordforms (as hypothesized by Zipf, 1949). Furthermore, both factors should

117 be most likely to produce homophones in exactly those regions of phonotactic space reported by

118 Piantadosi et al (2012): among short, phonotactically plausible wordforms.

119 It is currently unknown, however, how much homophony exists due to these simple,

120 distributional characteristics of languages alone. As a consequence, no evidence exists for or

121 against an efficiency-motivated direct pressure for homophony, as hypothesized by Zipf. The

122 current work asks two primary questions. First, to what extent is the amount of homophony

123 found in real human lexica attributable to indirect and uncontroversial factors such as length and

124 phonotactic regularities, without a direct pressure to reuse existing wordforms? And second, to 


\section{WHY DO HUMAN LANGUAGES HAVE HOMOPHONES?}

125 what extent are these indirect factors responsible for the concentration of homophony within

126 optimal regions of the lexicon?

127 To answer these questions, we constructed five series of artificial lexica, designed to

128 mirror the phonotactic regularities and word lengths of the real lexica of English, Dutch,

129 German, French, and Japanese. The generative model was an adaptation of the model used by

130 Dautriche et al (2017), in which a language's phonotactics were learned by training an $n$-phone

131 Markov Model on the set of unique wordforms in a lexicon. By observing the patterns of sounds

132 and sound combinations in a language, such a model can learn to encode phonotactic rules about

133 which sounds a word can start and end with, which sounds can occur in what sequence, and so

134 on. For each language, this model was then used to generate 10 artificial lexica, all matched for

135 the total number of words as well as the distribution of word lengths. For example, if the real

136 lexicon has 5,000 monosyllabic words, then each of the artificial lexica will also have 5,000

137 monosyllabic words. Furthermore, the distribution of sounds within and across those words will

138 approximate the phonotactics of the real language. These artificial lexica had no constraints

139 regarding homophones, reflecting a general tolerance for ambiguity; however, they also did not

140 contain a parameter biasing them toward the reuse of existing wordforms. Each artificial lexicon

141 thus represents one answer to the questions: 1) how much homophony can be expected to

142 emerge in a lexicon as a function of just the real, observed phonotactic regularities and the real,

143 observed distribution of word lengths; and 2) where should we expect to find the largest

144 concentrations of homophony as a function of these factors? They thus serve as a baseline

145 characterization of the effects of indirect causes of homophony. Comparing the real lexica to

146 these artificial ones reveals how much more or less homophony the real languages display —and 


\section{WHY DO HUMAN LANGUAGES HAVE HOMOPHONES?}

147 how much more or less concentrated it is - than would be expected without any direct pressure 148 for or against homophony ${ }^{2}$.

149 Note that these artificial lexica are not intended to serve as plausible models of lexicon

150 formation and change. Rather, as described above, they serve as statistical baselines in the

151 attempt to understand which theoretical parameters are necessary to explain the existence and

152 distribution of homophony in real lexica. For this reason, the artificial lexica are parameterized

153 solely by each particular language's phonotactics and distribution of word lengths.

154 The data and code to reproduce these analyses can be found on GitHub

155 (https://github.com/seantrott/homophone_simulations).

\section{Current Work}

\section{Materials and Methods}

158 Data. The English, German, and Dutch lexica were sourced from the CELEX lexical database

159 (Baayen et al, 1995). For French, we used the French Lexique (New et al, 2004). For Japanese, 160 we used the Japanese CallHome Lexicon (Kobayashi et al, 1996). We restricted our analysis to 161 lemma-only forms. Additionally, following Piantadosi et al (2012), we also excluded any words

162 containing spaces, hyphens, or apostrophes. This resulted in 41,887 entries for English (with

163 35,107 unique phonological forms), 51,719 entries for German (with 50,435 unique phonological

164 forms), 67,477 entries for Dutch (with 65,260 unique phonological forms), 47,782 entries for

165 French (with 37,278 unique phonological forms), and 51,147 entries for Japanese (with 40,449

\footnotetext{
${ }^{2}$ Note that our statistical models do not include a measure of frequency, even though this is included in the original model built in Piantadosi et al (2012). This is because it would not be meaningful to estimate frequency for the words in the artificial lexica.
} 


\section{WHY DO HUMAN LANGUAGES HAVE HOMOPHONES?}

166 unique phonological forms). As in Piantadosi et al (2012), words with multiple parts of speech

167 were counted as homophones ${ }^{3}$.

168 Methods.

Estimating number of syllables. Our primary determinant of word length was Number

170 of Syllables (or Number of Morae, in the case of Japanese; see below). While the real lexica

171 annotated this information for each lexical entry, it had to be estimated for the artificial lexica.

172 To ensure a fair comparison, we applied the same estimation procedure to wordforms in the real

173 lexica and wordforms in the artificial lexica.

174 For English, Dutch, German, and French, Number of Syllables was estimated by counting

175 the number of vowels occurring in a wordform's phonetic transcription. The set of possible

176 vowel characters for a given language was transcribed by hand and can be found on the project's

177 GitHub page. ${ }^{4}$

178 Since Japanese has been characterized as a mora-timed, rather than syllable-timed

179 language (Port et al., 1987), we calculated Number of Morae instead of Number of Syllables. In

180 addition to counting the number of vowels in a Japanese wordform, we counted the number of

181 nasal codas, as well geminate consonants (e.g., "kk” in Hokkaido, or "gg” in doggu). It should

182 be noted that the results we report below - both the replication of Piantadosi et al (2012), and the

183 comparison to the artificial lexica — are qualitatively similar whether word length in Japanese is

184 estimated using Number of Syllables or Number of Morae.

185 Counting number of homophones. Following Piantadosi et al (2012), we defined

186 Number of Homophones as the number of lexical entries with an identical phonological form as

\footnotetext{
${ }^{3}$ Importantly, this should only serve to inflate the estimated amount of homophony in naturally-occurring languages relative to the amount of homophony in the artificial lexica. Thus, it would actually work against the effects reported below (i.e., the artificial lexica exhibiting more homophony than the real lexica).

${ }^{4}$ Link: https://github.com/seantrott/homophone simulations
} 


\section{WHY DO HUMAN LANGUAGES HAVE HOMOPHONES?}

187 some target entry. ${ }^{5}$ This means the smallest possible value for Number of Homophones would be

188 (i.e., there are no other words with the same form in a given lexicon), and the largest possible

189 value would be one less than the size of the lexicon (i.e., all words share the same form).

190 After identifying the number of homophones for each entry in a lexicon, we reduced each

191 lexicon to the set of unique phonological wordforms (e.g., the 41,887 entries in English were

192 reduced to 35,107 unique forms).

193 Building the phonotactic model. In order to estimate the phonotactic plausibility of

194 wordforms in a lexicon, as well as to generate phonotactically plausible novel wordforms (see

195 below), it was first necessary to model the phonotactics of each language. We adapted the

196 procedure used in Dautriche et al (2017) ${ }^{6}$, which is described briefly below.

197 The phonotactics of a target language can be learned by observing, for all wordforms in

198 that language, which phonemes appear in what position and in what sequence. Specifically, an $n$ -

199 phone model calculates the probability of observing some phoneme in position $i$ given the

200 previous $n-1$ phonemes. For example, a 2-phone (biphone) model would condition the

201 probability of observing some phoneme as a function of the previous phoneme, i.e., $\mathrm{p}\left(\mathrm{X}_{\mathrm{i}} \mid \mathrm{X}_{\mathrm{i}-1}\right)$.

202 We included special symbols for the START and END of a word so that the model would also

203 learn which phonemes are most likely to begin and end a word in a given language. Note that

204 unlike Piantadosi et al (2012), these models were trained using the set of unique types (i.e.,

205 wordforms), rather than tokens (i.e., the actual instances of each wordform); this is because

206 training on tokens conflates phonotactic probability with frequency. This is analogous to the

207 main approach taken in Dautriche et al (2017).

\footnotetext{
${ }^{5}$ As pointed out by an anonymous reviewer, it is possible that the lexical resources we used, including CELEX, count as homophony some meanings that are actually polysemous. If this is the case, our estimates of homophony should actually be inflated for the real lexica, which would work against the effects reported below (i.e., the artificial lexica displaying higher incidences of homophony overall).

${ }^{6}$ Link to GitHub associated with Dautriche et al (2017): https://github.com/SbllDtrch/NullLexicons
} 


\section{WHY DO HUMAN LANGUAGES HAVE HOMOPHONES?}

While previous work (Dautriche et al, 2017) found that a 5-phone model effectively

209 captured phonotactic dependencies in English, Dutch, German, and French, we sought to

210 independently determine the optimal $n$ for each language, particularly because Japanese has

211 notably shorter syllables than the other four languages. To do this, we followed a similar

212 procedure as reported in Dautriche et al (2017) and Futtrell et al (2017). For each real lexicon,

213 we first extracted the set of unique wordforms (e.g., 35,107 wordforms in English), then

214 performed a series of train/test splits (75\% train, 25\% test). For each split, we trained a series of

$215 n$-phone models ranging from $n=1$ to $n=6$ on the wordforms in the training set, then evaluated

216 the probability of wordforms in the held-out test set. The basic motivation for this approach is as

217 follows: the optimal $n$-phone model for a language's phonotactics should be the model that,

218 when trained on a set of real wordforms, maximizes the probability of held-out wordforms that

219 also appear in that lexicon. Following Futtrell et al (2017), we ran a series of one-tailed two-

220 sample t-tests on the set of log-likelihoods of held-out wordforms obtained from each successive

221 n-phone model-i.e., the log-likelihoods obtained from the 2-phone model were compared to the

222 1-phone model, those from the 3-phone model were compared to the 2-phone model, and so on.

223 The optimal $n$ for a given lexicon was the smallest $n$ that represented a significant improvement

224 over the $n-1$ model for the same set of wordforms. Note that $\log _{10}$ was used to calculate $\log$

225 likelihoods (and subsequently, surprisal); the results are not qualitatively different when using $226 \log _{2}$ instead.

227 The mean log-likelihood calculated for held-out wordforms in each language are

228 visualized in Figure 1 below. Critically, we found that for English, Dutch, and German, the 5-

229 phone model represented a significant improvement over the 4-phone model. That is, held-out

230 wordforms were significantly more likely under the 5-phone model than the 4-phone model for 


\section{WHY DO HUMAN LANGUAGES HAVE HOMOPHONES?}

231 English $(t=4.05, p<.001)$, Dutch $(t=3.55, p<.001)$, and German $(t=7.31, p<.001)$.

232 However, the 6-phone model either did not improve or actually decreased model fit (suggesting

233 overfitting) in each language (all $t \leq 0)$. The 4-phone model was optimal for French $(t=8.67, p$

$234<.001)$ and Japanese $(t=4.08, p<.001)$. Thus, a 5-phone model was used to evaluate the

235 probabilities of wordforms in English, Dutch, and German (and generate artificial lexica for

236 those languages), and a 4-phone model was used for French and Japanese.

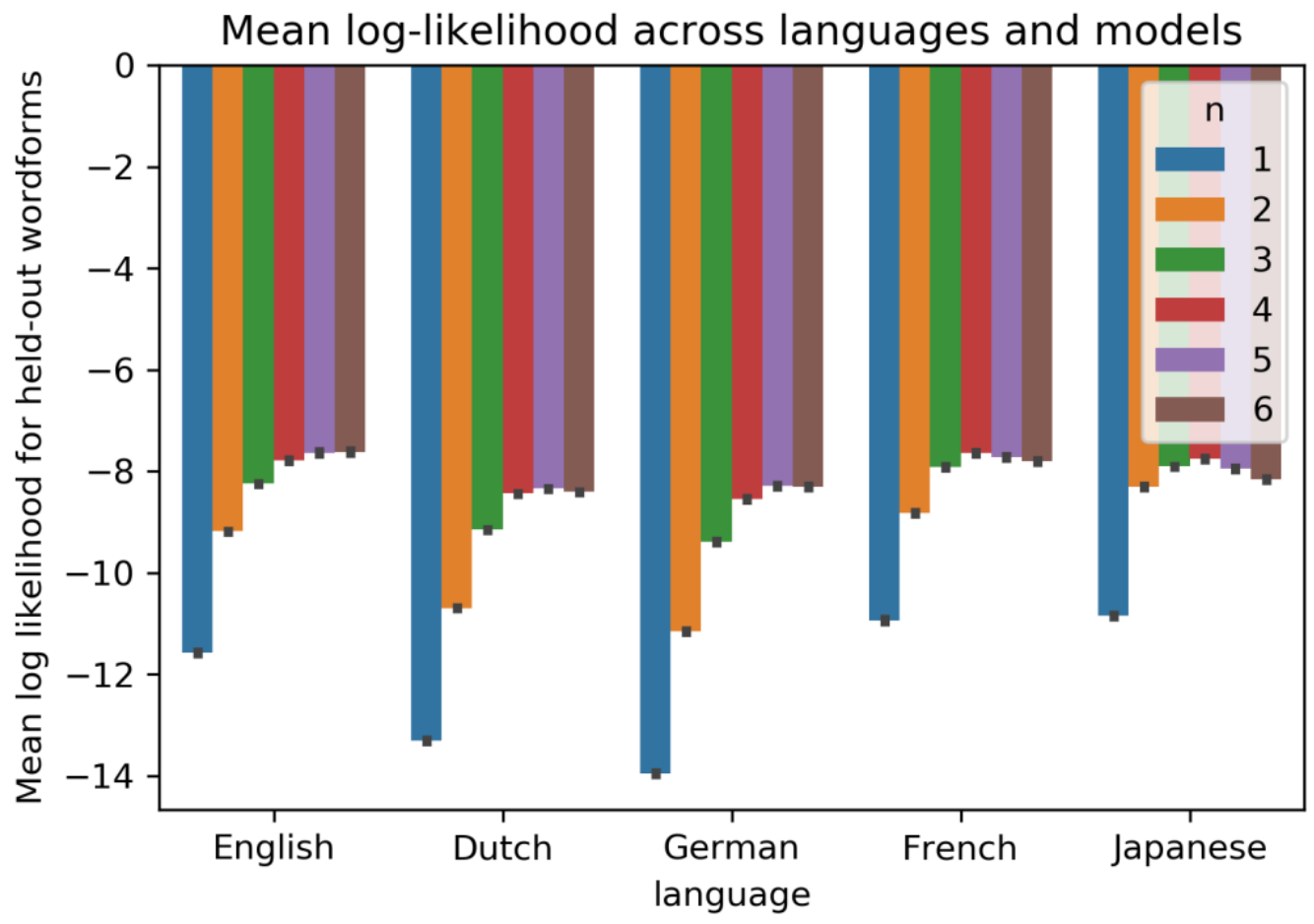

Figure 1: Mean log-likelihood of held-out wordforms for each n-phone model, across languages. Higher values (i.e., less negative) indicate higher probability under that model. For English, Dutch, and German, increasing n up to 5 significantly improved model fit over the 4-phone model; a 6-phone model did not improve fit. For French and Japanese, a 4-phone model was the highest $\mathrm{n}$ representing an improvement over the (n-1) model.

This model allows us to evaluate the probability of a given wordform, which can be

243 defined as the product of all the transitional probabilities between each phoneme in that

244 wordform (including the start and end symbols). The Surprisal of a given wordform is thus

245 defined as the negative log probability of observing that particular sequence of phonemes:

246 Surprisal $($ word $)=-\log (\mathrm{p}($ word $))$. As in Piantadosi et al (2012), we normalized this measure to the 


\section{WHY DO HUMAN LANGUAGES HAVE HOMOPHONES?}

247 number of phonemes in a word to ensure that surprisal could be compared across words of

248 different length: Normalized Surprisal = Surprisal(word) $/$ Length(word).

249 Once the model was built for each language, it was then used to generate novel

250 wordforms in an iterative manner. For each word, the model began with the START symbol,

251 then generated a phoneme conditioned on that start symbol (i.e., one of the phonemes likely to

252 occur at the beginning of the word). The next phoneme was then conditioned on the first

253 phoneme and the START symbol, and so on, until the model produced the END symbol,

254 signaling the end of the word.

255 Finally, as in Dautriche et al (2017), we assigned non-zero probability to unobserved

256 phoneme sequences using an identical smoothing procedure; they report that "optimal smoothing

257 was obtained with Laplace smoothing with parameter .01" (pg. 132), so this was the value we

258 used in configuring the phonotactic model.

Generating artificial lexica. We generated 10 artificial lexica for each real lexicon.

261 First, we identified the number of words in the real lexicon, as well as the distribution of their

262 lengths, as measured by Number of Syllables (see above for the estimation procedure). Each

263 artificial lexicon was constrained to have the same overall number of words (not wordforms) as

264 the corresponding real lexicon, as well as the same distribution of word lengths. For example,

265 since the real English lexicon has 7706 monosyllabic words, each artificial English lexicon was

266 also constrained to have 7706 monosyllabic words.

267 We then built a phonotactic model for the real lexicon as described above, and used this

268 model to generate wordforms for each artificial lexicon. For each potential wordform, we

269 estimated the Number of Syllables to determine whether to add it to the artificial lexicon-e.g., if 


\section{WHY DO HUMAN LANGUAGES HAVE HOMOPHONES?}

270 the word had 1 syllable and the artificial lexicon still had fewer monosyllabic words than the real

271 lexicon, the word was added to the lexicon; otherwise, it was discarded. No other constraints

272 were placed on the generation of wordforms; we allowed the model to generate real wordforms,

273 as well as wordforms that were homophonous with wordforms already in the lexicon. This

274 process continued until the artificial lexicon had the same number of words of each length as the 275 real lexicon.

276 Note that the models used to generate the artificial lexica were trained on the entire set of

277 unique wordforms for the target lexicon; however, qualitatively similar results were obtained

278 using a 50/50 split of the target lexicon to generate and evaluate wordform phonotactic

279 probability (see Supplementary Analysis 5).

$281 \quad$ Results

282 Replication and extension of previous findings.

283 First, we replicated the primary analysis reported by Piantadosi et al (2012) on the real

284 lexica of English, Dutch, and German, and extended this analysis to two non-Germanic

285 languages: French and Japanese. Using a Poisson regression, we asked whether a wordform's

286 \#Homophones (the number of additional, distinct meanings) was related to its length in syllables

287 (\#Syllables) and its phonotactic plausibility (Surprisal). As in Piantadosi et al (2012), we used

288 the Normalized Surprisal measure described above, obtained by dividing a wordform's Surprisal

289 by its length in phones. 


\section{WHY DO HUMAN LANGUAGES HAVE HOMOPHONES?}

We found significant, negative relationships in the real lexica between \#Homophones and \#Syllables (or \#Morae ${ }^{7}$ in Japanese) for English $[\beta=-0.72, \mathrm{SE}=0.03, \mathrm{p}<.001]$, German $[\beta=-$ $0.69, \mathrm{SE}=.04, \mathrm{p}<.001]$, Dutch $[\beta=-1.11, \mathrm{SE}=0.03, \mathrm{p}<.001]$, French $[\beta=-0.35, \mathrm{SE}=0.02, \mathrm{p}$ $<.001]$, and Japanese $[\beta=-1.01, \mathrm{SE}=0.01, \mathrm{p}<.001]$. That is, for all five languages, shorter wordforms were more likely to have more homophones-consistent with the notion that lexica recycle short wordforms for multiple meanings.

However, we found positive ${ }^{8}$ relationships between Normalized Surprisal and

297 \#Homophones across all real languages but Japanese, i.e., less phonotactically plausible

298 wordforms (as measured by a 5-phone model or 4-phone model, as appropriate) were more likely

to have more homophones. This was true for English $[\beta=0.78, \mathrm{SE}=0.03, \mathrm{p}<.001]$, German $[\beta$ $=0.86, \mathrm{SE}=0.06, \mathrm{p}<.001]$, Dutch $[\beta=0.997, \mathrm{SE}=0.04, \mathrm{p}<.001]$, French $[\beta=0.73, \mathrm{SE}=$ $0.04, \mathrm{p}<.001]$, but not Japanese $[\beta=0.0004, \mathrm{SE}=0.031, \mathrm{p}=.99]$. This is in contrast to the original result reported by Piantadosi et al (2012), who found a negative relationship between Normalized Surprisal and \#Homophones in German and Dutch. Piantadosi et al (2012). First, while Piantadosi et al (2012) used a 3-phone model to determine phonotactic plausibility, we used 4-phone and 5-phone models to estimate wordform probability, which were found to improve model fit over a 3-phone model (see Figure 1). Second, our models were trained using lexical types, as opposed to tokens (which would conflate frequency with phonotactic probability). And third, our estimates were not calculated using held-out

\footnotetext{
${ }^{7}$ Like syllables, a mora is a unit of timing, and is usually considered the basis of the sound system in Japanese. A single mora in Japanese is constituted by a vowel (or an onset and a vowel); nasal codas also constitute a separate mora, as does the first part of a geminate consonant.

${ }^{8}$ Note that negative relationships were obtained between the non-normalized Surprisal measure and Number of Homophones across each language; these results are described in Supplementary Analysis 2. However, this nonnormalized Surprisal measure conflates phonotactic plausibility with word length, which is why Normalized Surprisal may be a better measure overall.
} 


\section{WHY DO HUMAN LANGUAGES HAVE HOMOPHONES?}

310 wordforms, as they were in Piantadosi et al (2012). This final explanation is explored in

311 Supplementary Analysis 3; using 10-fold cross-validation to obtain our surprisal estimates, we

312 found that the coefficients for Normalized Surprisal were closer to 0 for all the real lexica, and

313 negative in Japanese. Thus, a likely reason for the disparity is that the surprisal estimates given

314 here were not calculated using held-out wordforms.

315 However, the central question of the current work concerns the comparison between the

316 real and artificial lexica. The results of these comparisons are described in detail below, both

317 concerning the amount of homophony across the real and artificial lexica, as well as where those

318 homophones are concentrated.

319 Simulated lexica exhibit higher upper-bounds on homophony.

320 We operationalized the amount of homophony in three ways. First, we measured the

321 Maximum Number of Homophones per wordform - that is, in a given lexicon, how many

322 homophones does the most homophonous wordform have? Second, we measured the Mean

323 Number of Homophones per wordform. And third, we measured Homophony Rate: how many

324 wordforms in a lexicon have at least 1 homophone? In all cases, more positive values reflect a

325 greater amount of homophony. For each measure in each language, we compared the distribution

326 of values obtained from the simulated lexica to the value in the real lexicon. This enabled us to

327 ask the question: to what extent can the amount of homophony in a language be attributed to a

328 selective pressure for lexical ambiguity, as opposed to an emergent outcome of a language's

329 phonotactics and distribution of word lengths? Note that for all of these measures, the values

330 obtained for the real and artificial lexica were significantly different ${ }^{9}(p<.001)$, except where

331 noted otherwise.

\footnotetext{
${ }^{9}$ Significance was determined by comparing a given test statistic for the real lexicon $t_{\text {real }}$ to the corresponding distribution of test statistics obtained from the artificial lexica, $\mathrm{T}_{\text {artificial }}$. Each of these values was centered according
} 


\section{WHY DO HUMAN LANGUAGES HAVE HOMOPHONES?}

Across all five languages, the simulated lexica had a significantly larger Maximum

333 Number of Homophones on a single wordform (see Figure 2 below). For example, the most

334 homophonous wordforms in the real English lexicon had at most 7 homophones, while the most

335 homophonous wordforms in the simulated English lexica had anywhere from 17 to 28

336 homophones $(\mathrm{M}=19.8, \mathrm{SD}=3.3)$. This difference was particularly stark for Dutch: the most

337 homophonous wordform in the Dutch lexicon had 5 homophones, while the maximum number of 338 homophones per wordform in the simulated lexica ranged from 72 to $116(\mathrm{M}=97.1, \mathrm{SD}=$

339 15.13).

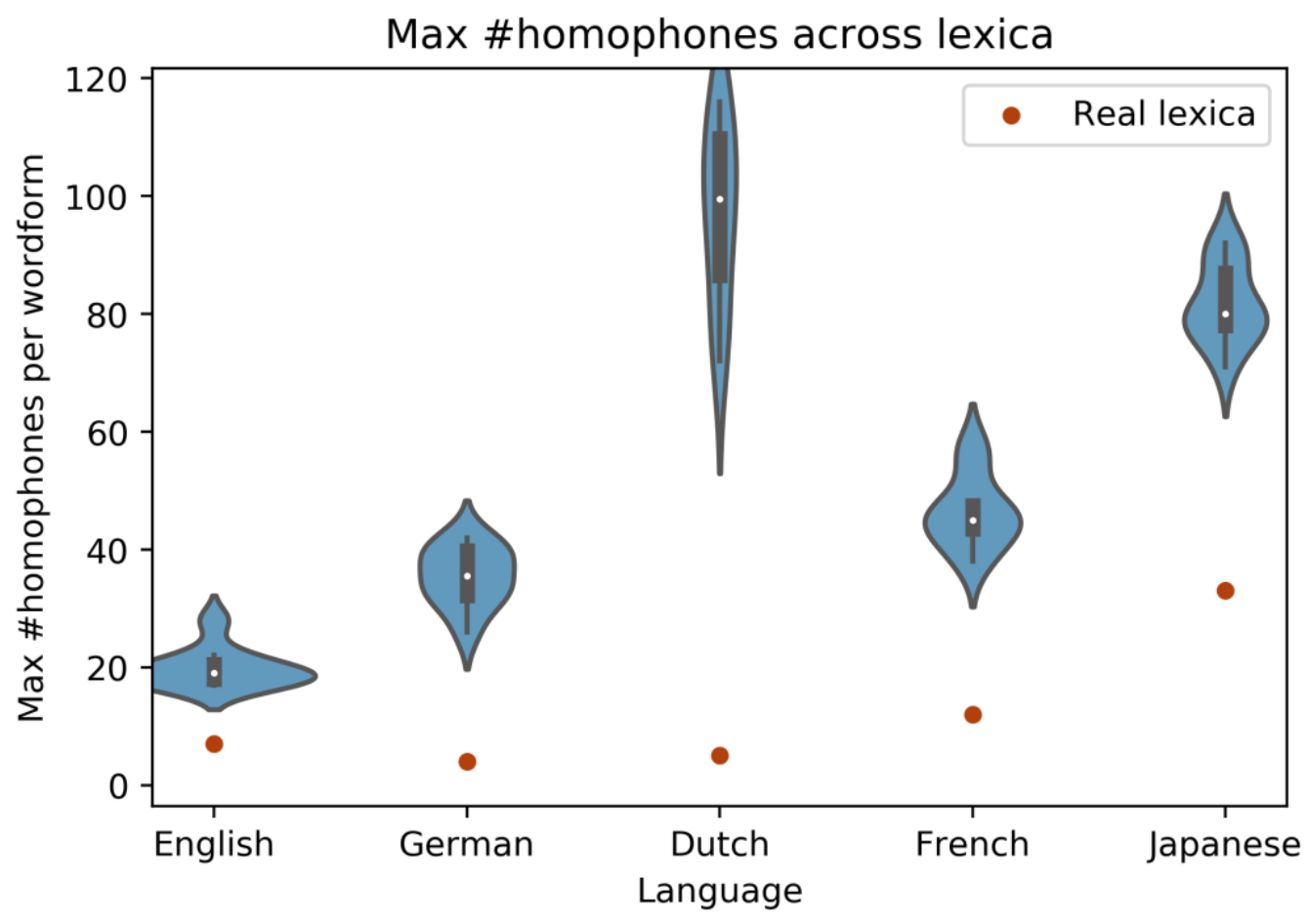

to the mean of $\mathrm{T}_{\text {artificial }}$, denoted here as $T_{\text {, }}^{\prime}{ }_{\text {arificial }}$ and $t^{\prime}$ real . We then conducted a two-tailed significance test, i.e., calculating the proportion of values in $\mid \mathrm{T}^{\prime}$ artificial $\mid$ that were greater than or equal to $\mid t^{\prime}$ real $\mid$. This proportion corresponds to a p-value; e.g., if all the values in $\left|\mathrm{T}^{\prime}{ }_{\text {artificial }}\right|$ are less than $\mid t^{\prime}$ real $\mid, p=0$. 


\section{WHY DO HUMAN LANGUAGES HAVE HOMOPHONES?}

Figure 2: For each language, the most homophonous wordforms in the artificial lexica (shown by the vioin plots) have more homophones than the most homophonous wordforms in the real lexica (shown by the orange dots). The artificial lexica uniformly exhibit a higher upper-bound (Maximum Number of Homophones) on homophony.

As expected, there was considerable variability across the five languages in how much homophony was tolerated per wordform. For example, the real Japanese lexicon exhibited a much higher upper-bound on homophony (33) than the real German lexicon (4); this is not surprising, given the limited syllable inventory of Japanese (on the order of 100 possible syllables) relative to German (over 1000 possible syllables, conservatively). Importantly, however, the simulated Japanese lexica still had more homophones per wordform than their real counterpart, ranging from 71 to $92(\mathrm{M}=81.6, \mathrm{SD}=6.67)$. In other words, despite inter-linguistic variability, the simulated lexica in each language all exhibited higher upper-bounds on how much homophony was tolerated for a given wordform - the most homophonous wordforms were considerably more ambiguous, sometimes by an order of magnitude (e.g., in Dutch).

Similarly, with the exception of Japanese $(p=.5)$, wordforms in the simulated lexica had a significantly larger Mean Number of Homophones than wordforms from their real counterparts (see Figure 3 below for an illustration); in Japanese, the Mean Number of Homophones per wordform was at least as high in the artificial lexica as it was in the real lexicon. ${ }^{10}$ For example, wordforms in English have on average 0.19 homophones; in contrast, the average number of homophones per wordform in the simulated English lexica ranged from 0.22 to $0.23(\mathrm{M}=0.22$, $\mathrm{SD}=0.003)$. Again, there was considerable inter-linguistic variability; wordforms in the real Japanese lexicon have more homophones on average (0.26) than wordforms in the real German lexicon (0.02). However, in each language, the average number of homophones per wordform

\footnotetext{
${ }^{10}$ Note that for Japanese, the Mean Number of Homophones per wordform is actually higher in the artificial lexica than the real lexicon with the use of a 5-phone model, rather than a 4-phone model.
} 


\section{WHY DO HUMAN LANGUAGES HAVE HOMOPHONES?}

364 was at least as large in the simulated lexica as the real counterparts - and for four of the five

365 languages, wordforms in the simulated lexica were, on average, more ambiguous than those in

366 the real lexica.

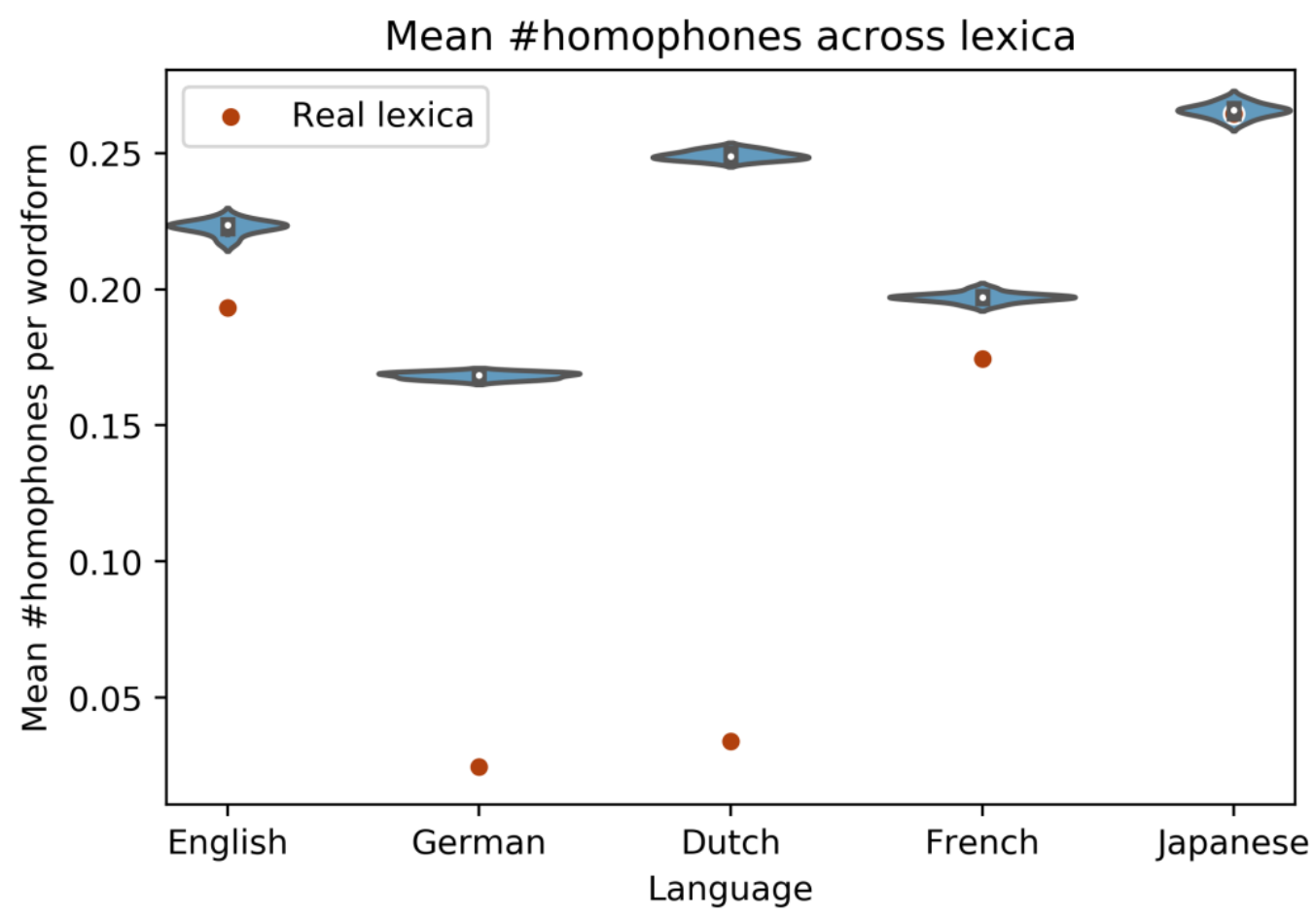

368 Figure 3: In every language but Japanese, wordforms in the artificial lexica (shown by violin plots) have more homophones

369 (Mean Number of Homophones) on average than wordforms in the real lexica (shown by orange dots). In Japanese, the Mean

370 Number of Homophones per wordform is at least as high in the artificial lexica $(M=0.27, S D=0.002)$ as the real lexica (.26).

371 The results for the Homophony Rate (i.e., the proportion of wordforms with at least one

372 homophone) across real and simulated lexica were more mixed (see Figure 4 below). 


\section{WHY DO HUMAN LANGUAGES HAVE HOMOPHONES?}

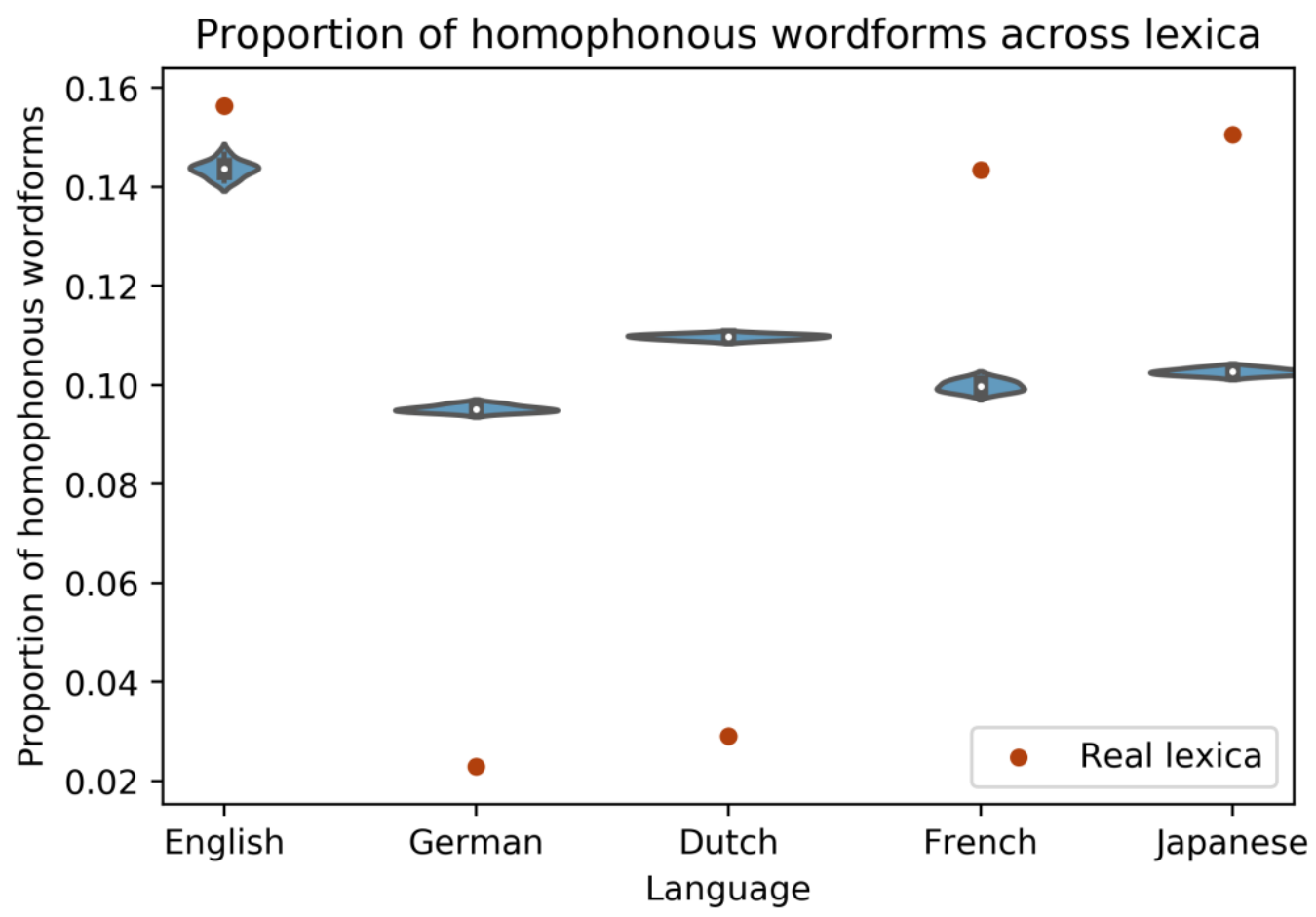

373

374 Figure 4: The artificial Dutch and German have a higher proportion of wordforms with at least one homophone (shown by the

375 violin plots) than their real counterparts (shown by the orange dots). However, the artificial French, Japanese, and English

376 artificial lexica have lower Homophony Rates than the real lexica.

378 In two languages (German and Dutch), the simulated lexica had significantly more

379 homophonous wordforms, sometimes by a factor of $2 \mathrm{x}$ or $3 \mathrm{x}$; for example, the homophony rate

380 in the real Dutch lexicon was 0.03 , while the rate in the simulated lexica ranged from 0.108 to

$3810.11(\mathrm{M}=.11, \mathrm{SD}=.0004)$. On the other hand, the Homophony Rate in the real English lexicon

382 (0.156) was significantly higher than the rate the simulated lexica $(\mathrm{M}=0.143, \mathrm{SD}=.002)$;

383 similarly, the Homophony Rate for the real French and Japanese lexica were significantly higher

384 than that for the artificial lexica.

385 Together, these results suggest that the amount of homophony in the five real lexica is

386 not the result of a direct pressure for ambiguity. In fact, the real lexica actually display less

387 homophony than the artificial ones in some measures, particularly the upper-bound of 


\section{WHY DO HUMAN LANGUAGES HAVE HOMOPHONES?}

388 homophones tolerated for a given wordform and the mean number of homophones per

389 wordform. This means that merely the pressure for highly probable phonotactic sequences,

390 combined with the observed distribution of word lengths, can produce concentrations of

391 homophony in a lexicon that are as dense or denser than in real lexica, without a direct pressure

392 to recycle entire wordforms.

\section{Simulated lexica exhibit more efficient reuse of optimal wordforms.}

394 We then asked whether homophones were more concentrated in optimal regions of phonotactic

395 space in the simulated lexica or their real counterparts. That is, to what extent do the

396 phonotactics of a language, as well as its distribution of word lengths, account for the finding

397 that more optimal wordforms tend to have more homophones?

398 In order to assess the degree to which homophony was optimally distributed in a lexicon,

399 we regressed a wordform's \#Homophones against two operationalizations of wordform

400 optimality: its length (\#Syllables) and its phonotactic plausibility (Normalized Surprisal). For

401 each lexicon, we extracted the following information from the model: 1) pseudo- $\mathrm{R}^{2}$, as a measure

402 of overall model fit; 2) the coefficient for \#Syllables; and 3) the coefficient for Normalized

403 Surprisal. A larger, more positive value for (1) reflects more efficient reuse overall, and more

404 negative values for (2) and (3) reflect more efficient reuse along those particular dimensions of

405 wordform optimality. Then, for each language, we compared each of these test statistics from the

406 real lexicon to the distribution of test statistics obtained from the corresponding simulated lexica.

407 The significance for each of these comparisons was assessed in the same way as above. All of

408 the comparisons described revealed significant difference. To preview the overall finding, in all

409 cases, the simulated lexica exhibited stronger effects (i.e., more optimally distributed

410 wordforms) than their real counterparts. 


\section{WHY DO HUMAN LANGUAGES HAVE HOMOPHONES?}

Across all five languages, the distribution of pseudo- $\mathrm{R}^{2}$ values obtained from the

412 simulated lexica were significantly higher than the pseudo- $\mathrm{R}^{2}$ value from the real lexicon (see

413 Figure 5 below). Pseudo-R ${ }^{2}$ reflects a model's goodness-of-fit, i.e., how well the predictors in a

414 model explain variance in the dependent variable. Thus, this indicates that two

415 operationalizations of wordform optimality — its length, and its phonotactic plausibility—were

416 better predictors of homophony across all of the simulated lexica than their real counterparts, for

417 each language. For example, the pseudo- $\mathrm{R}^{2}$ for the model constructed on the real English lexicon

418 was .143, while the mean for the simulated lexica was $0.17(\mathrm{SD}=.004)$. Some differences were

419 even starker: the pseudo- $\mathrm{R}^{2}$ for the real German lexicon was .09, while the distribution of

420 pseudo- $\mathrm{R}^{2}$ values for the simulated German lexica averaged more than twice that $(\mathrm{M}=0.231, \mathrm{SD}$

$421=.003)$. Concretely, this means that homophony is better predicted by wordform optimality in

422 the artificial than real lexica.

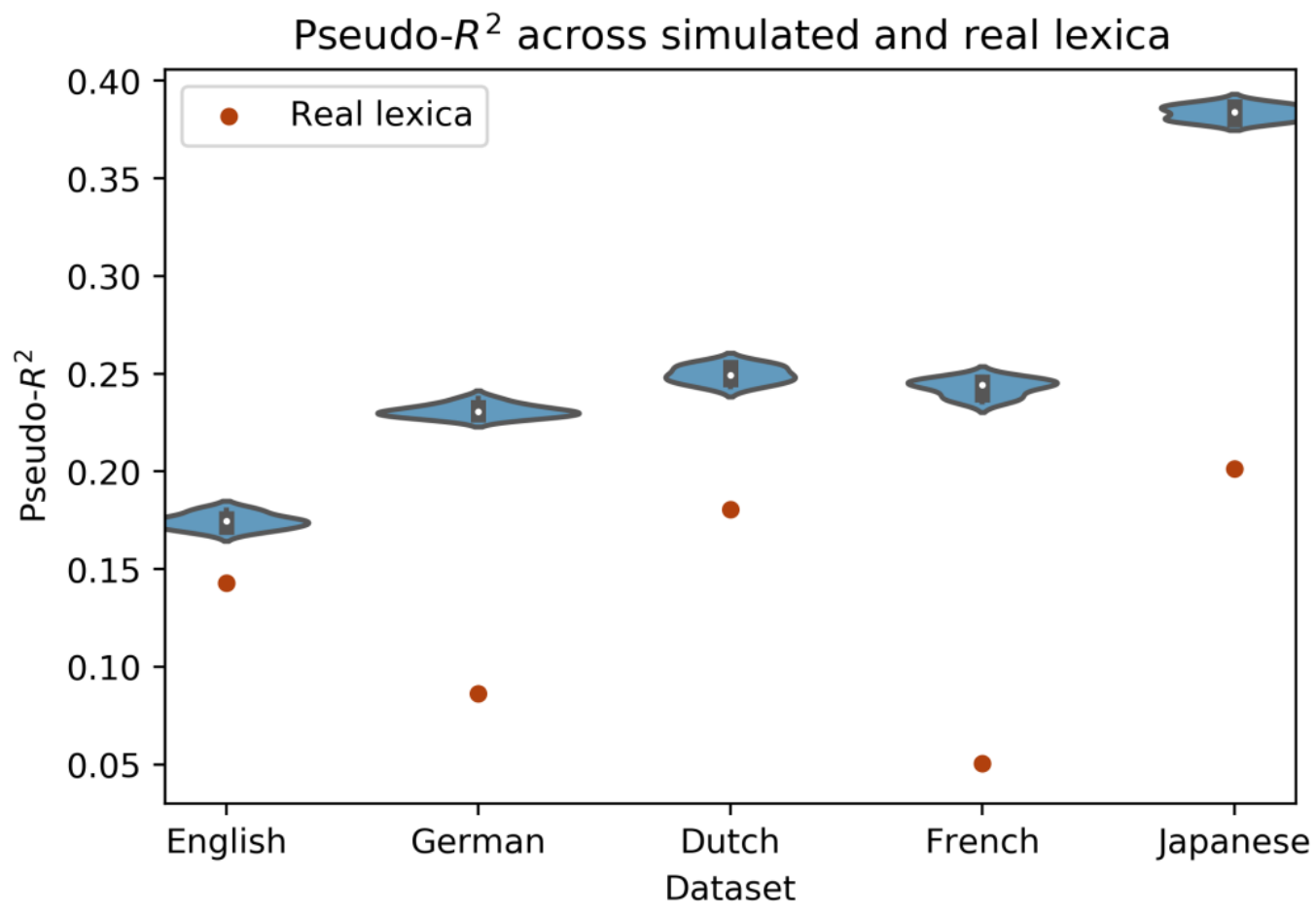

423 


\section{WHY DO HUMAN LANGUAGES HAVE HOMOPHONES?}

Figure 5: We built a series of Poisson regression models predicting \#Homophones from \#Syllables and Normalized Surprisal. In 425 each language, the models constructed for the artificial lexica (shown by violin plots) exhibit better model fit (larger pseudo- $R^{2}$ )

426 than the models constructed for the real lexicon (shown by orange dots).

Further evidence comes from direct comparison of the coefficients for both predictors

429 (Number of Syllables and Surprisal) across the real and artificial lexica. As reported earlier, the

430 real lexica all exhibited negative relationships between Number of Syllables and Number of

431 Homophones - i.e., short wordforms have more homophones in all five languages. However, the 432 simulated lexica exhibited significantly stronger relationships, as depicted in Figure 6 below.

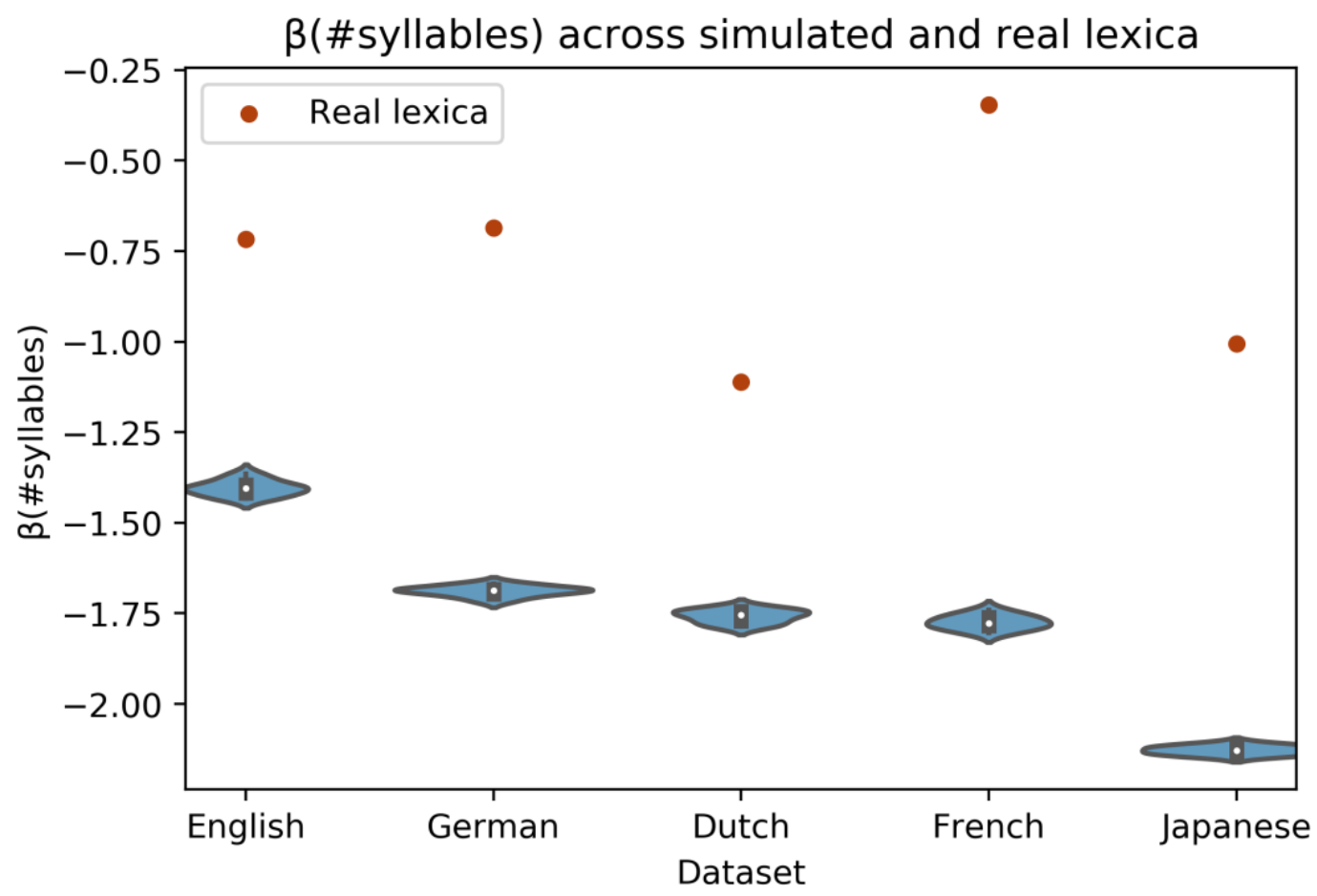

434 Figure 6: Word length (as measured in \#Syllables) is a better predictor of homophony in the artificial lexica (shown by the violin 435 plots) than the real lexica (shown by orange dots).

437 For example, the coefficient for Number of Syllables in the real English lexicon was -0.717 , but

438 the coefficients for the simulated English lexica were approximately twice as large $(\mathrm{M}=-1.4, \mathrm{SD}$

$439=.02$ ). In some cases, the difference was even larger, as in French: here, the coefficients for the 


\section{WHY DO HUMAN LANGUAGES HAVE HOMOPHONES?}

440 simulated lexica $(\mathrm{M}=-1.77, \mathrm{SD}=.02)$ were approximately five times as large as the coefficient

441 for the real lexicon $(-.35)$.

442 Even more striking results were obtained for Surprisal: the real lexica actually exhibited

443 positive relationships between Surprisal and Number of Homophones, while the artificial lexica

444 all demonstrated negative relationships (see Figure 7); these differences were significant for each

445 language. In other words, the artificial lexica reused short, phonotactically plausible wordforms

446 to a greater extent than did their real counterparts.

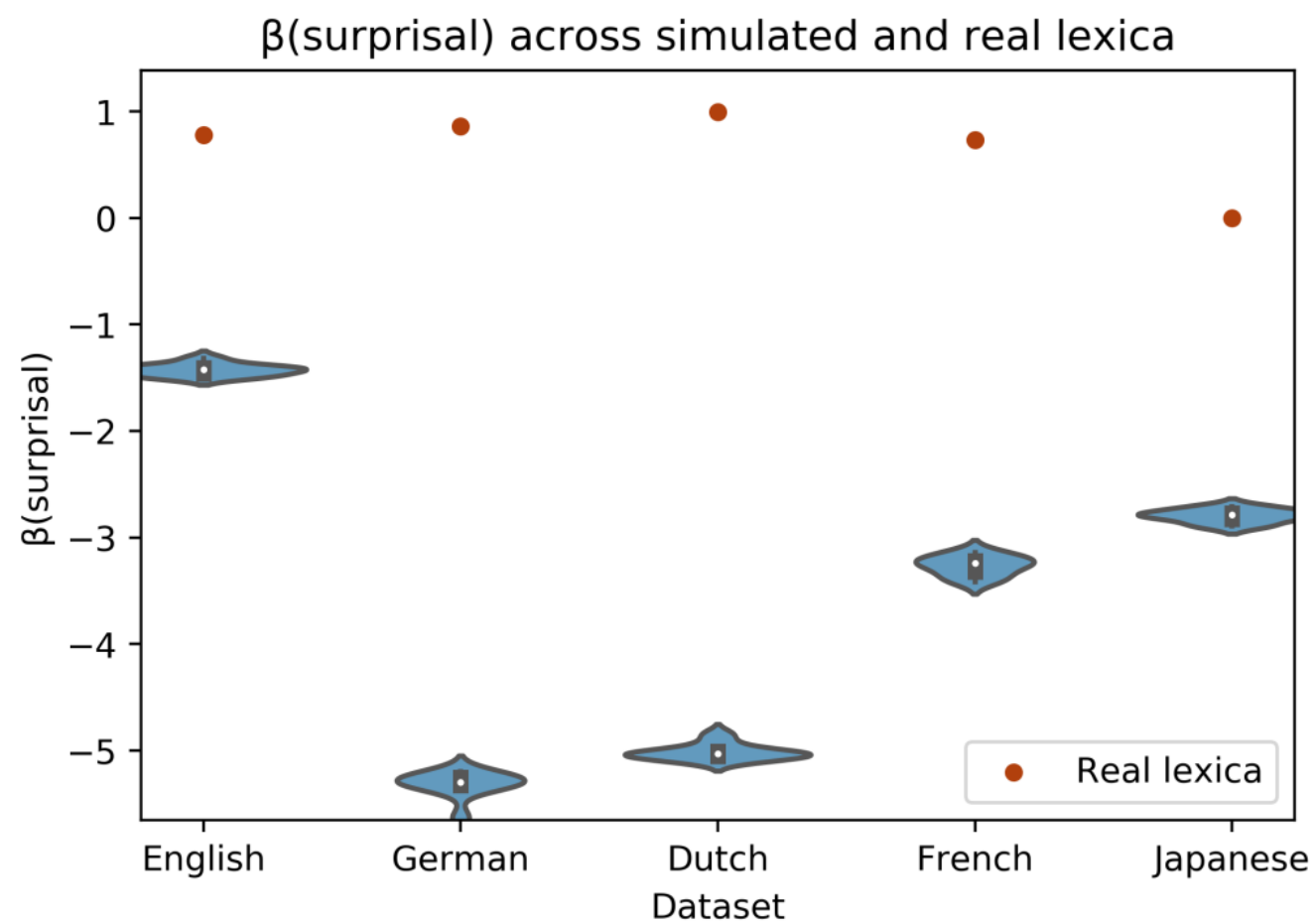

447

448 Figure 7: Phonotactic Surprisal was more negatively correlated with Number of Homophones (i.e., more probable wordforms

449 had comparatively more homophones) in the artificial lexica (shown by violin plots) than real lexica (shown by orange dots). 


\section{WHY DO HUMAN LANGUAGES HAVE HOMOPHONES?}

453 In the current work, we asked whether the prevalence of homophony across five languages-

454 English, German, Dutch, French, and Japanese - could be plausibly attributed to a direct pressure

455 to recycle optimal wordforms. We reasoned that even without a direct pressure for ambiguity, an

456 absence of a pressure against ambiguity should result in some amount of homophony in a

457 lexicon, simply as a result of a language's phonotactic constraints and the distribution of words

458 across different lengths. Under this view, the selective pressure is for well-formed phonotactic

459 sequences as opposed to entire wordforms; the pressure to use well-formed sequences could

460 result in homophony, particularly for the most phonotactically probable wordforms.

461 Furthermore, given that the proportion of occupied phonotactic space will always be highest for

462 short wordforms, homophony should also be most likely to occur in short words.

463 We tested this view by simulating a series of artificial lexica for each of the five

464 languages. Across all five languages, we found that wordforms in the real lexica had either fewer

465 or an equivalent number of homophones on average as wordforms in the artificial lexica (in

466 every language but Japanese, wordforms in the artificial lexica were more ambiguous on average

467 than those in the real lexica (see Figure 2)). The real lexica also uniformly exhibited lower

468 upper-bounds on the number of homophones tolerated per wordform (see Figure 3). This was

469 true despite considerable cross-linguistic variability in the propensity towards homophony

470 overall (e.g., Japanese vs. Dutch); in each language, the artificial lexica surpassed their real

471 counterparts in terms of the degree to which a wordform could be saturated with many meanings.

472 The main exception to this trend was Homophony Rate (the proportion of wordforms with at

473 least one homophone): for English, French, and Japanese (but not German and Dutch), the real

474 lexica had higher Homophony Rates than the artificial lexica. This will be discussed in more

475 detail below. Finally, statistical analyses of where these homophones were distributed revealed 


\section{WHY DO HUMAN LANGUAGES HAVE HOMOPHONES?}

476 that homophones in the real lexica were concentrated less efficiently in "optimal" regions of

477 phonotactic space: across all languages, word length and phonotactic plausibility—taken as

478 operationalizations of wordform optimality - were better predictors of homophony in the

479 artificial lexica than the real lexica (see Figures 5-7).

480 There are two conclusions to be drawn from these results. First, neither the amount of

481 homophony in these five real languages, nor the apparent concentration of homophones among

482 optimal regions of phonotactic space, requires explanation by a direct pressure to recycle entire

483 wordforms. Rather, homophony appears to be a natural and perhaps inevitable consequence of

484 other features of a language-i.e., its phonotactics and distribution of word lengths. Of course,

485 these features may themselves be related to efficiency, as noted in the Introduction-but

486 indirectly so.

487 Second, real lexica may actually be the product of a pressure against homophony. The

488 artificial lexica were modeled using only two parameters: the phonotactics of the target lexicon

489 and a particular distribution of word lengths. They were not designed to explicitly select for

490 homophony, nor did they contain a parameter selecting against homophony. In other words, they

491 reflect the consequence of allowing the phonotactics of a language to determine its space of

492 realized wordforms, under the assumption that the speakers of that language place no upper limit

493 on how many homophones are tolerated per wordform. This resulted in considerably more

494 homophones per wordform than observed in real languages. For example, wordforms in the real

495 Dutch lexicon had at most 5 homophones, whereas the average upper-bound in the Dutch lexica

496 was 97-more than 16 times as high. Furthermore, homophony in the artificial lexica was more

497 likely to be found among more optimal wordforms. 


\section{WHY DO HUMAN LANGUAGES HAVE HOMOPHONES?}

One explanation for this result is that real lexica are subject to a pressure against

oversaturating the same wordform with too many unrelated meanings - no matter how "optimal"

500 it is. Clearly this pressure is not absolute: homophony does still exist (to varying degrees) in real

501 languages - and in fact, some languages (French, English, and Japanese) had a higher proportion

502 of wordforms with at least one homophone than their artificial counterparts. This suggests that

503 the pressure is not against the existence of homophony per se, but rather, could reflect a

504 constraint on the extent to which any given wordform can be saturated with distinct, unrelated

505 meanings. Assigning too many unrelated meanings to the same signal could impede

506 communication or learning (Casenhiser, 2005; though see Dautriche et al, 2018), and may thus

507 be selected against. Such a pressure against oversaturation is roughly analogous to what others

508 have termed diversification (Zipf, 1949) or a pressure for clarity (Piantadosi et al, 2012).

509 However, unlike Zipf (1949), we find no opposing pressure towards unification; instead,

510 homophony appears to emerge naturally as a function of other pressures (e.g., phonotactics), and

511 is attenuated in particular wordforms (i.e., it does not reach the potential predicted by that

512 wordform's phonotactics) due to a pressure against oversaturation.

513 There are a number of explanations for how this direct or indirect pressure against over-

514 saturation might come about. For example, the attenuation of homophony could manifest as a

515 kind of smoothing of high-probability phoneme sequences across phonological neighborhoods as

516 opposed to being concentrated in a specific wordform. (A wordform's neighborhood is the set of

517 wordforms differing from it in only one phoneme.) This could satisfy the pressure to reuse well-

518 formed phonotactic sequences while also avoiding potential impediments to communication

519 caused by overloading the same high-probability wordform with too many meanings. 


\section{WHY DO HUMAN LANGUAGES HAVE HOMOPHONES?}

This account leads to testable predictions. If real lexica are subject to this smoothing

521 process, they should have larger phonological neighborhoods than the artificial lexica, which

522 were placed under no pressure against ambiguity. Indeed, previous work using an identical

523 generative model (Dautriche et al, 2017) found exactly this: across four languages (English,

524 German, Dutch, and French), real lexica exhibit more "clumpiness" (i.e., larger and more

525 densely connected neighborhoods) than ought to be expected merely as a function of those

526 languages' phonotactics. We extended a subset of their analyses to the set of artificial lexica we

527 constructed, counting as "neighbors" any two wordforms that could be converted into each other

528 via one phoneme substitution, deletion, or insertion (Luce \& Pisoni, 1998; Vitevitch \& Luce,

529 1999; Dell \& Gordon, 2003). Under this definition of neighbor, the neighbors of the word cat

530 would include rat (substitution), at (deletion), and cast (insertion). Consistent with prior work,

531 and despite a different operationalization of neighborhoods from Dautriche et al (2017), we

532 found that wordforms in the real lexica had larger average neighborhood sizes than wordforms in

533 the artificial lexica (see Figure 8 below). For example, wordforms in the real English lexica

534 averaged 2.56 neighbors, whereas the mean neighborhood sizes in the artificial English lexica

535 ranged from 2.23 to $2.32(\mathrm{M}=2.28, \mathrm{SD}=0.03)$. This result is the inverse of our finding

536 regarding homophony_-wordforms in the artificial lexica have more homophones on average

537 than wordforms in the real lexica. In other words, the artificial lexica appear to optimize for

538 dense concentrations of homophony, while the real lexica appear to optimize for larger

539 neighborhoods. This apparent trade-off can also be illustrated by comparing both the rank-

540 distribution of homophone counts and rank-distribution of neighborhood sizes across the real and

541 artificial lexica (see Figure 9). 


\section{WHY DO HUMAN LANGUAGES HAVE HOMOPHONES?}

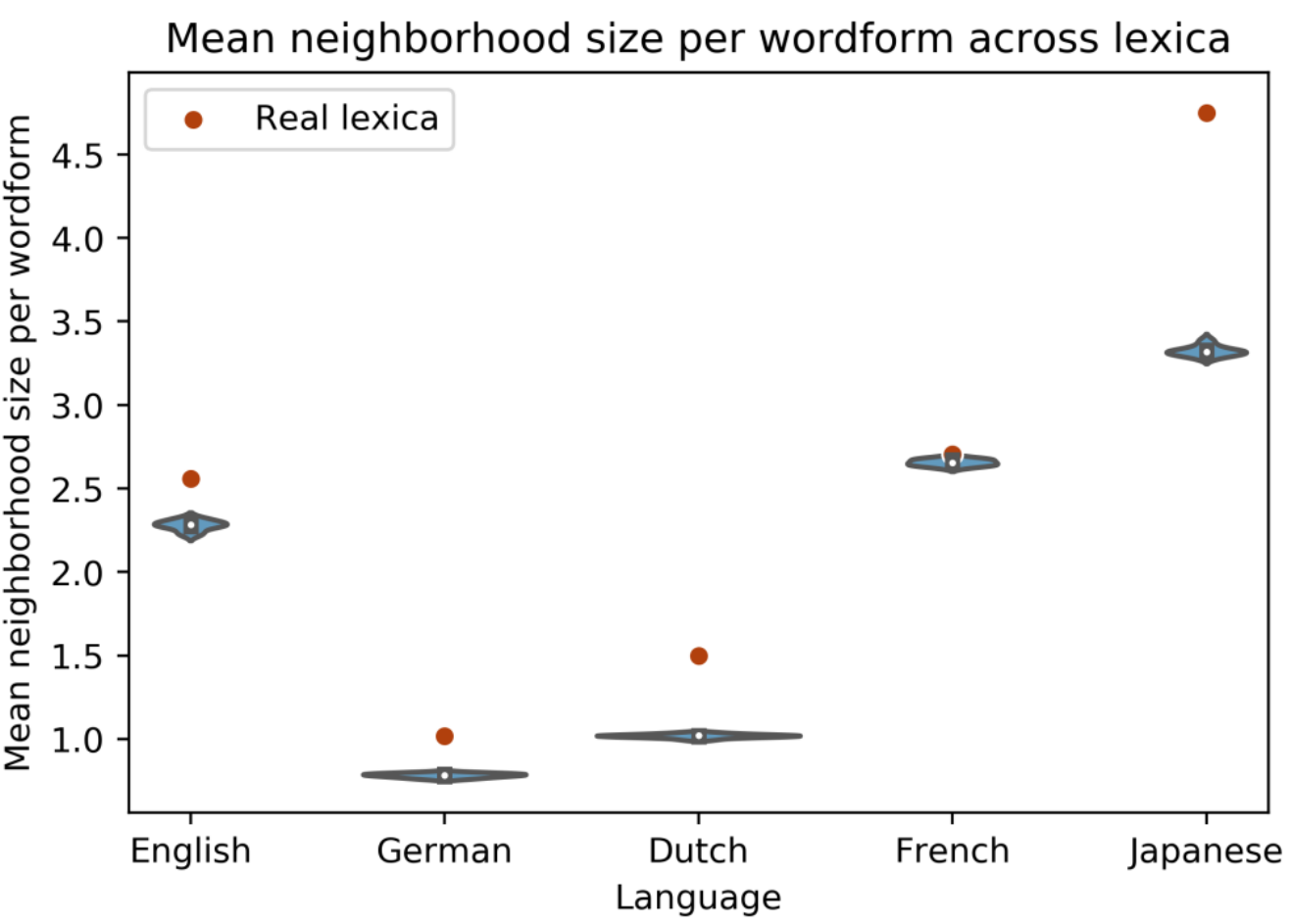

543 Figure 8: Consistent with previous work (Dautriche et al, 2017), wordforms in the real lexica (shown by orange dots) have

544 larger lexical neighborhoods (i.e., the set of words differing in exactly one phoneme) on average than wordforms in the artificial

545 lexica (shown by violin plots). Note that this is true even in French, where the values are closest: wordforms in the real French

546 lexicon have 2.71 neighbors on average, whereas wordforms in the artificial lexica have approximately 2.66 neighbors on

547 average $(S D=.02)$.

548 


\section{WHY DO HUMAN LANGUAGES HAVE HOMOPHONES?}

550

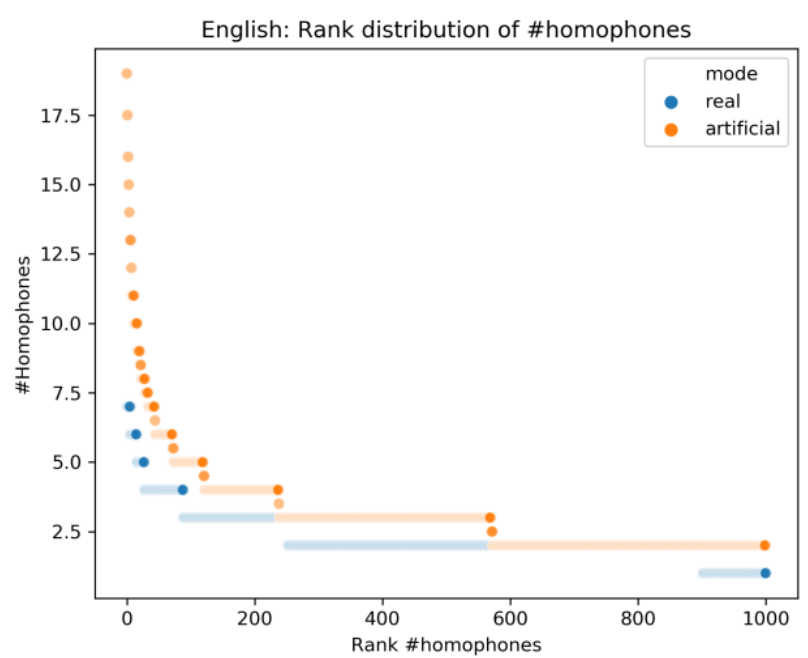

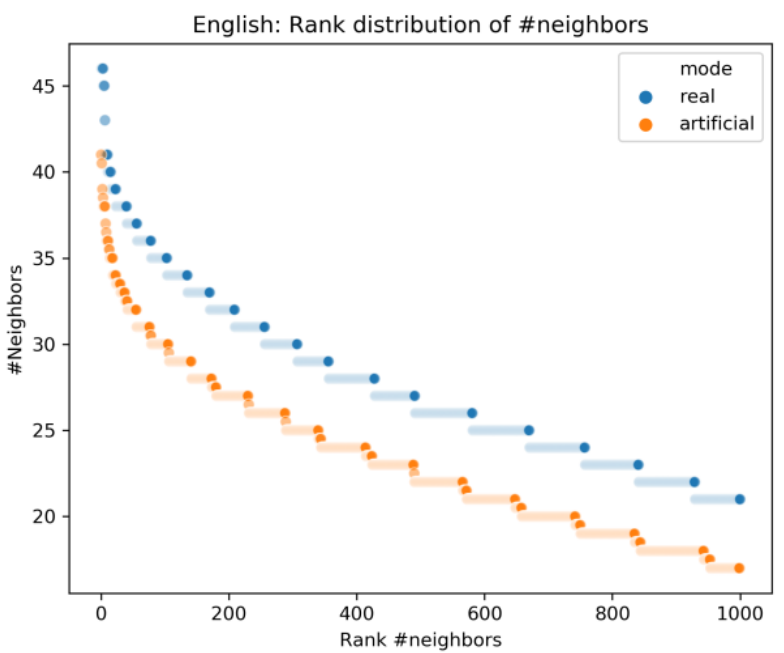

551

Figure 9: Rank-distribution of homophone counts (left) and rank-distribution of neighborhood sizes (right) across the real and artificial English lexica. The most homophonous wordforms in the artificial lexica are more homophonous than equivalently ranked wordforms in the real lexica. Conversely, the wordforms with the largest neighborhoods in the artificial lexica still have smaller neighborhoods than equivalently ranked wordforms in the real lexica.

Taken together, these findings are broadly consistent with the hypothesis that real lexica could be subject to a pressure against oversaturating the same wordform with too many meanings, and this selection against homophony could instead result in the creation of lexical neighbors. Of course, a similar effect could be achieved not through selection against high levels of homophony but rather from a positive pressure towards large neighborhoods, i.e., a "clumpy" lexicon. As Dautriche et al (2017) argue, dense lexical neighborhoods may have many beneficial consequences, e.g., for word learning (Coady \& Aslin, 2003; Storkel et al, 2006; though see also Swingley \& Aslin, 2007) and word production (Vitevitch et al, 2002; Vitevitch \& Sommers, 2003). It is impossible to know from the current work whether the disparity between the real and artificial lexica is due to a direct pressure in real lexica against oversaturation that results in dense neighborhoods, or a positive selection for dense neighborhoods that results in less homophony. Future work could explore this potential trade-off at both the psychological level of 


\section{WHY DO HUMAN LANGUAGES HAVE HOMOPHONES?}

568 explanation (e.g., whether learners make errors when learning homophones that lead to the

569 creation of near neighbors), and by simulating such pressures during the lexicon generation

570 process (e.g., whether a direct pressure in favor of large neighbors reduces the number of

571 homophones, or whether a direct pressure against over-saturation increases neighborhood size).

572 Homophones could conceivably be reduced in real lexica through other, more indirect

573 mechanisms as well. Notably, many human languages have rich morphological structure,

574 allowing them to flexibly combine existing morphemes to construct novel meanings. While the

575 real lexica we analyzed excluded wordforms derived via inflectional morphology, they did not

576 exclude derivational morphology (e.g., adding the suffix -ify to the adjective humid creates the

577 verb humidify; adding the suffix -ness to the adjective happy creates the noun happiness).

578 Morphological compositionality allows speakers to convey new meanings without coining

579 entirely new wordforms - but it also avoids the need to reuse existing wordforms for new,

580 unrelated meanings (i.e., homophony). Thus, compositionality represents an efficient mechanism

581 for recycling existing lexical materials that also avoids outright ambiguity. Clearly, wordforms in

582 the artificial lexica were not constructed via processes of morphological composition. Future

583 work could also explore whether parameterizing these artificial lexica according to the

584 morphology of the underlying real lexicon would decrease the overall homophony, and if so,

585 how. (See Supplementary Analysis 4 for further exploration of the relationship between

586 derivational morphology and homophony in real lexica.)

587 In addition to real lexica exhibiting a lower upper-bound on homophony overall, we

588 found that their homophones were less optimally distributed - that is, homophones were much

589 more concentrated among short, phonotactically likely wordforms in the artificial lexica than in

590 their real counterparts. This result is surprising on its face: why do real lexica apparently prefer 


\section{WHY DO HUMAN LANGUAGES HAVE HOMOPHONES?}

591 (at least relative to the phonotactic baselines) to distribute their homophones across less optimal

592 regions of the lexicon? Even if real lexica select against over-saturation, intuition suggests that

593 the homophones that are preserved should be concentrated among short, phonotactically likely

594 wordforms. One possible explanation for this result is that the pressures that ordinarily select

595 against homophony are reduced for longer wordforms - there are at least two accounts as to why

596 this may be the case. The first account is that longer wordforms might be more contextually

597 discriminable than short wordforms and are thus more likely to be preserved in the lexicon. If

598 this is true, the distinct senses of homophonous wordforms should be better disambiguated by

599 contextual cues (e.g., some representation of the linguistic context) for longer wordforms. The

600 second account holds that because longer wordforms are comparatively less common than short

601 wordforms, they require less frequent disambiguation. Even if longer wordforms are no more

602 contextually discriminable than short wordforms, they are encountered less often. If a frequent

603 need to disambiguate is one of the factors that selects against homophony-e.g., because

604 disambiguation may incur processing costs, no matter how marginal-homophones should be

605 relatively more likely to be preserved among infrequent wordforms than frequent ones. Note that

606 this does not predict that short wordforms have less homophones overall; past work (Piantadosi

607 et al, 2012) has shown empirically that this is not the case. Rather, the penalty against accruing

608 multiple meanings will be proportionately less for longer, less frequent wordforms. Therefore,

609 less frequent wordforms should experience less of a reduction in their projected homophony

610 (relative to their phonotactics) than more frequent ones.

611 As noted above, the artificial lexica are intended as statistical baselines to determine

612 which theoretical parameters are required to explain homophony, not as models of the many

613 other pressures that real lexica are subject to. Thus, our work does not elucidate the 


\section{WHY DO HUMAN LANGUAGES HAVE HOMOPHONES?}

614 developmental or historical mechanisms by which homophones arise, nor the processes by which

615 they might be selected against or be preserved in a lexicon. There are a number of known sources

616 of homophony in real lexica, including sound change and lexical borrowing (Ogura \& Wang,

617 2006; Ke, 2007). Despite some debate about the extent to which homophony-generating sound

618 changes are avoided (Sampson, 2013; Wedel et al, 2013; Sampson, 2015; Yin \& White, 2018),

619 there are many attested examples of phoneme losses and mergers resulting in homophony, such

620 as knight and night in English, or as a consequence of the many phoneme mergers experienced in

621 Middle Chinese (Ke, 2007; Sampson, 2013; Sampson, 2015). Similarly, lexical borrowing can

622 lead to homophony; for example, the English words sheik and chic were both borrowed from

623 different languages at different time points (16 ${ }^{\text {th }}$ century Arabic vs. $19^{\text {th }}$ century French,

624 respectively), and both have an identical phonological form (Ke, 2007). A satisfying explanation

625 of homophony at a mechanistic level should incorporate these generative processes-i.e., the

626 mutations by which potential homophones are introduced into a lexicon. Such a model should

627 also predict which potential homophones will be selected against (and what form this selection

628 process takes, i.e., whether it is via the avoidance of homophony-inducing mergers (Wedel et al,

629 2013; Yin \& White, 2018) or something else) and which will be preserved. Homophones should

630 be more likely to survive in a lexicon if their meanings are systematically made sufficiently

631 discriminable by context (Dautriche et al, 2018). A better understanding of this process would

632 also yield insights into which sources of contextual information human speakers and

633 comprehenders routinely sample and deploy for disambiguation, and therefore influence

634 language change.

635 We began by asking why a system that appears to be optimized for efficient

636 communication (Gibson et al, 2019) contains apparently inefficient properties such as lexical 


\section{WHY DO HUMAN LANGUAGES HAVE HOMOPHONES?}

637 ambiguity. A series of simulations suggests no evidence for a direct selection pressure in favor of

638 homophones. Rather, the concentration of homophony among short, high-probability wordforms

639 can be explained purely as a function of a language's phonotactics and distribution of word

640 lengths, which perhaps themselves are the result of a pressure for efficiency. In fact, real lexica

641 may even select against dense concentrations of homophony. We have suggested one

642 mechanism: they might "smooth out" high-probability phonotactic sequences across lexical

643 neighborhoods instead of concentrating these sequences in a single wordform. The product is

644 lexica that are slightly less optimal in phonotactic terms but may better conform to other

645 requirements of humans who need to use them.

\section{Acknowledgments}

648 We are very grateful to Tamara Rhodes for helping to secure access to the Japanese CallHome

649 Lexicon. We also thank Steven Piantadosi for helping us understand the operationalization of 650 surprisal in his model, Pamela Rivière for her help in concatenating the figures, Sarah Creel for

651 advice on relevant literature about lexical neighborhoods in language acquisition, and Isabelle

652 Dautriche for making the code for learning and generating phonotactic sequences available on

653 GitHub. We are grateful to the anonymous reviewers for their advice both on the theoretical

654 framing of the paper, as well as the construction and evaluation of the phonotactic models.

655 Finally, we thank members of the Center for Research in Language and the Language and

656 Cognition Lab at UC San Diego for their helpful comments on earlier versions of these analyses. 


\section{WHY DO HUMAN LANGUAGES HAVE HOMOPHONES?}

658

659

660

661

662

663

664

665

666

667

668

669

670

671

672

673

674

675

676

677

678

679

\section{References}

Aina, L., Gulordava, K., \& Boleda, G. (2019). Putting words in context: LSTM language models and lexical ambiguity. arXiv preprint arXiv:1906.05149.

Baayen, R. H., Piepenbrock, R., \& Gulikers, L. (1995). The CELEX lexical database (release 2). Distributed by the Linguistic Data Consortium, University of Pennsylvania.

Casenhiser, D. M. (2005). Children's resistance to homonymy: An experimental study of pseudohomonyms. Journal of Child Language, 32(2), 319-343.

Coady, J. A., \& Aslin, R. N. (2003). Phonological neighbourhoods in the developing lexicon. Journal of Child language, 30(2), 441-469.

Coady, J. A., \& Aslin, R. N. (2004). Young children's sensitivity to probabilistic phonotactics in the developing lexicon. Journal of Experimental Child Psychology, 89(3), 183-213.

Dautriche, I. (2015). Weaving an ambiguous lexicon (Doctoral dissertation, Sorbonne Paris Cité).

Dautriche, I., Mahowald, K., Gibson, E., Christophe, A., \& Piantadosi, S. T. (2017). Words cluster phonetically beyond phonotactic regularities. Cognition, 163, 128-145.

Dautriche, I., Fibla, L., Fievet, A. C., \& Christophe, A. (2018). Learning homophones in context: Easy cases are favored in the lexicon of natural languages. Cognitive psychology, 104, $83-105$.

Dell, G. S., \& Gordon, J. K. (2003). Neighbors in the lexicon: Friends or foes. Phonetics and phonology in language comprehension and production: Differences and similarities, 6, 937.

Ferreira, V. S. (2008). Ambiguity, accessibility, and a division of labor for communicative success. Psychology of Learning and motivation, 49, 209-246. 


\section{WHY DO HUMAN LANGUAGES HAVE HOMOPHONES?}

680 Futrell, R., Mahowald, K., \& Gibson, E. (2015). Large-scale evidence of dependency length minimization in 37 languages. Proceedings of the National Academy of Sciences, 112(33), 10336-10341.

Futrell, R., Albright, A., Graff, P., \& O’Donnell, T. J. (2017). A generative model of phonotactics. Transactions of the Association for Computational Linguistics, 5, 73-86.

Gathercole, S. E., Willis, C., Emslie, H., \& Baddeley, A. D. (1991). The influences of number of syllables and wordlikeness on children's repetition of nonwords. Applied psycholinguistics, 12(3), 349-367.

Gibson, E., Futrell, R., Jara-Ettinger, J., Mahowald, K., Bergen, L., Ratnasingam, S., Gibson, M., Piantadosi, S.T. and Conway, B.R. (2017). Color naming across languages reflects color use. Proceedings of the National Academy of Sciences, 114(40), pp.10785-10790.

Gibson, E., Futrell, R., Piandadosi, S. T., Dautriche, I., Mahowald, K., Bergen, L., \& Levy, R. (2019). How Efficiency Shapes Human Language. Trends in cognitive sciences.

Holle, H., \& Gunter, T. C. (2007). The role of iconic gestures in speech disambiguation: ERP evidence. Journal of cognitive neuroscience, 19(7), 1175-1192.

695 Holler, J., \& Beattie, G. (2003). Pragmatic aspects of representational gestures: Do speakers use them to clarify verbal ambiguity for the listener?. Gesture, 3(2), 127-154.

697 Jusczyk, P. W., Luce, P. A., \& Charles-Luce, J. (1994). Infants' sensitivity to phonotactic patterns in the native language. Journal of memory and Language, 33(5), 630.

699 Ke, J. (2006). A cross-linguistic quantitative study of homophony. Journal of Quantitative Linguistics, 13(01), 129-159.

701 Kemp, C., \& Regier, T. (2012). Kinship categories across languages reflect general 702 communicative principles. Science, 336(6084), 1049-1054. 


\section{WHY DO HUMAN LANGUAGES HAVE HOMOPHONES?}

703 Kemp, C., Xu, Y., \& Regier, T. (2018). Semantic typology and efficient communication. Annual $704 \quad$ Review of Linguistics, 4, 109-128.

705 Kidd, E., \& Holler, J. (2009). Children's use of gesture to resolve lexical ambiguity. 706 Developmental Science, 12(6), 903-913.

707 Levinson, S. C. (2000). Presumptive meanings: The theory of generalized conversational $708 \quad$ implicature. MIT press.

709 Luce, P. A., \& Pisoni, D. B. (1998). Recognizing spoken words: The neighborhood activation $710 \quad$ model. Ear and hearing, 19(1), 1.

711 Mahowald, K., Dautriche, I., Gibson, E., \& Piantadosi, S. T. (2018). Word forms are structured 712 for efficient use. Cognitive science, 42(8), 3116-3134.

713 Munson, B. (2001). Phonological pattern frequency and speech production in adults and 714 children. Journal of Speech, Language, and Hearing Research.

715 New, B., Pallier, C., Brysbaert, M., \& Ferrand, L. (2004). Lexique 2: A new French lexical 716 database. Behavior Research Methods, Instruments, \& Computers, 36(3), 516-524.

717 Ogura, M., \& Wang, W. S. (2006). Ambiguity and language evolution: evolution of homophones 718 and syllable number of words.

719 Piantadosi, S. T., Tily, H. J., \& Gibson, E. (2009). The communicative lexicon hypothesis. In 720 The 31st annual meeting of the Cognitive Science Society (CogSci09) (pp. 2582-2587).

721 Piantadosi, S. T., Tily, H., \& Gibson, E. (2011). Word lengths are optimized for efficient communication. Proceedings of the National Academy of Sciences, 108(9), 3526-3529.

723 Piantadosi, S. T., Tily, H., \& Gibson, E. (2012). The communicative function of ambiguity in 724 language. Cognition, 122(3), 280-291. 


\section{WHY DO HUMAN LANGUAGES HAVE HOMOPHONES?}

725 Port, R. F., Dalby, J., \& O’Dell, M. (1987). Evidence for mora timing in Japanese. The Journal 726 of the Acoustical Society of America, 81(5), 1574-1585.

727 Regier, T., Kay, P., \& Khetarpal, N. (2007). Color naming reflects optimal partitions of color 728 space. Proceedings of the National Academy of Sciences, 104(4), 1436-1441.

729 Regier, T., Carstensen, A., \& Kemp, C. (2016). Languages support efficient communication 730 about the environment: Words for snow revisited. PloS one, 11(4), e0151138.

731 Rodd, J., Gaskell, G., \& Marslen-Wilson, W. (2002). Making sense of semantic ambiguity: Semantic competition in lexical access. Journal of Memory and Language, 46(2), 245266.

734 Sampson, G. (2013). A counterexample to homophony avoidance. Diachronica, 30(4), 579-591.

735 Sampson, G. (2015). A Chinese phonological enigma. Journal of Chinese Linguistics, 43(2), $736 \quad 679-691$.

737 Storkel, H. L., Armbrüster, J., \& Hogan, T. P. (2006). Differentiating phonotactic probability and neighborhood density in adult word learning. Journal of Speech, Language, and Hearing Research.

740 Swingley, D., \& Aslin, R. N. (2007). Lexical competition in young children's word learning. $741 \quad$ Cognitive psychology, 54(2), 99-132.

742 Vitevitch, M. S., \& Luce, P. A. (1999). Probabilistic phonotactics and neighborhood activation in 743 spoken word recognition. Journal of Memory and Language, 40(3), 374-408.

744 Vitevitch, M. S. (2002). The influence of phonological similarity neighborhoods on speech 745 production. Journal of Experimental Psychology: Learning, Memory, and Cognition, $28(4), 735$. 


\section{WHY DO HUMAN LANGUAGES HAVE HOMOPHONES?}

747 Vitevitch, M. S., \& Sommers, M. S. (2003). The facilitative influence of phonological similarity and neighborhood frequency in speech production in younger and older adults. Memory \& cognition, 31(4), 491-504.

Wasow, T., Perfors, A., \& Beaver, D. (2005). The puzzle of ambiguity. Morphology and the web of grammar: Essays in memory of Steven G. Lapointe, 265-282.

752 Wedel, A., Kaplan, A., \& Jackson, S. (2013). High functional load inhibits phonological contrast loss: A corpus study. Cognition, 128(2), 179-186.

754 Winters, J., Kirby, S., \& Smith, K. (2018). Contextual predictability shapes signal autonomy. Cognition, 176, 15-30.

756 Xu, Y., \& Regier, T. (2014). Numeral systems across languages support efficient communication: From approximate numerosity to recursion. In Proceedings of the

Yin, S. H., \& White, J. (2018). Neutralization and homophony avoidance in phonological learning. Cognition, 179, 89-101.

761 Zaslavsky, N., Kemp, C., Regier, T., \& Tishby, N. (2018). Efficient compression in color naming and its evolution. Proceedings of the National Academy of Sciences, 115(31), 7937-7942.

763 Zipf, G. (1949). Human behavior and the principle of least effort. New York: Addison-Wesley. 
WHY DO HUMAN LANGUAGES HAVE HOMOPHONES?

770

771

772

773

774 


\section{WHY DO HUMAN LANGUAGES HAVE HOMOPHONES?}

\section{Supplementary Materials}

\section{Supplementary Analysis 1: 2-phone model}

778 To ensure that the observed amounts and concentrations of homophony in the simulated lexica

779 were not due to the 5-phone phonotactic model overfitting, we also constructed a 2-phone model

780 on the real English lexicon using an otherwise identical procedure, and generated an additional

78110 artificial lexica. The Maximum Number of Homophones for the newly generated artificial

782 lexica was even higher than those created by the 5-phone model $(\mathrm{M}=434.3, \mathrm{SD}=26.272)$; this

783 was in turn over 60x larger than the upper-bound for the real English lexicon (7 homophones).

784 For both the real and artificial lexica, we then regressed a wordform's Number of

785 Homophones against its Number of Syllables and Normalized Surprisal. Surprisal was now

786 calculated using the bi-phone model for both the real and artificial English lexica. We compared

787 the goodness-of-fit of the models constructed on the artificial vs. real English lexica using the

788 pseudo- $\mathrm{R}^{2}$ metric. Consistent with the results reported in the main text, the models built on the

789 artificial English lexica had considerably higher pseudo- $\mathrm{R}^{2}$ values $(\mathrm{M}=0.304, \mathrm{SD}=0.004)$ than

790 the model built on the real English lexicon (0.13). Additionally, the coefficient values for

791 Number of Syllables were larger in magnitude for the artificial lexica $(\mathrm{M}=-2.54, \mathrm{SD}=.03)$ than

792 the real (-0.94). Finally, the coefficient values for Normalized Surprisal were larger in the 793 expected direction for the artificial lexica $(\mathrm{M}=-1.05, \mathrm{SD}=.11)$ than the real $(0.1)$.

794 These supplementary results provide additional evidence that the real English lexicon

795 actually demonstrates less efficient reuse than one would expect simply as a result of its

796 phonotactics and distribution of word lengths. A model with weaker assumptions about the range 


\section{WHY DO HUMAN LANGUAGES HAVE HOMOPHONES?}

797 of phonotactic dependencies in English (i.e., 2 phones vs. 5 phones) still produced dense

798 concentrations of homophony among the shortest, most phonotactically plausible wordforms.

799

800 


\section{WHY DO HUMAN LANGUAGES HAVE HOMOPHONES?}

801 Supplementary Analysis 2: Non-normalized Surprisal in the real lexica. We conducted a

802 secondary analysis using non-normalized Surprisal (i.e., before dividing by the number of

803 phones in a wordform) and \#Syllables as regressors. We found negative relationships between

804 Surprisal and \#Homophones in all five languages: English $[\beta=-0.2, \mathrm{SE}=.03, \mathrm{p}<.001]$, German

$805[\beta=-0.98, \mathrm{SE}=.05, \mathrm{p}<.001]$, Dutch $[\beta=-0.77, \mathrm{SE}=.03, \mathrm{p}<.001]$, French $[\beta=-0.32, \mathrm{SE}=$

$806.01, \mathrm{p}<.001]$, and Japanese $[\beta=-0.8, \mathrm{SE}=.01, \mathrm{p}<.001]$

807 These results are more consistent with the notion that real lexica recycle more

808 phonotactically plausible wordforms for multiple meanings. However, non-normalized Surprisal

809 is confounded with word length, which is why Normalized Surprisal may ultimately be a better

810 measure of phonotactic plausibility.

811 Critically, identical models built on the artificial lexica still exhibited much better model

812 fit, as measured by pseudo-R ${ }^{2}$ (see Supplementary Figure 1 below); furthermore, non-normalized

813 Surprisal exhibited stronger, more negative relationships with Number of Homophones in the

814 artificial lexica than the real lexica (see Supplementary Figure 2). However, as depicted in

815 Supplementary Figure 3, using non-normalized Surprisal in the model resulted in more positive

816 coefficients for Number of Syllables for the artificial lexica, likely because non-normalized

817 Surprisal and word length are highly collinear, perhaps particularly for the artificial lexica.

818 Importantly, though, regardless of whether Surprisal or Normalized Surprisal is used as a

819 measure of phonotactic plausibility, the artificial lexica exhibit stronger relationships between

820 phonotactic plausibility and Number of Homophones than their real counterparts — suggesting

821 that wordforms are more heavily concentrated among optimal regions of phonotactic space in the 822 artificial lexica. 


\section{WHY DO HUMAN LANGUAGES HAVE HOMOPHONES?}

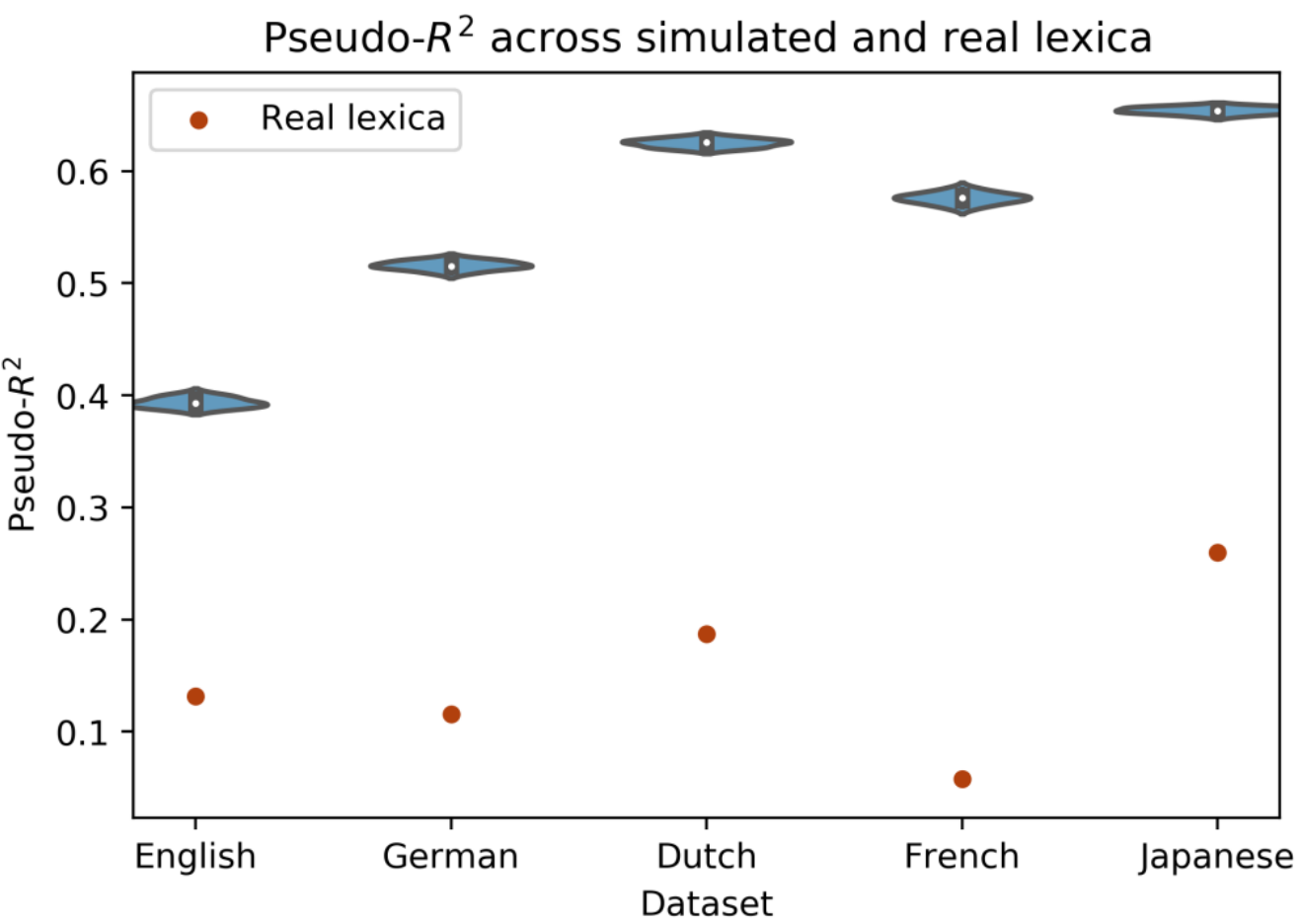

824

Dataset

825 Supplementary Figure 1: Pseudo- $R^{2}$ values were higher for all of the artificial lexica than their real counterparts, indicating that

826 Number of Syllables and Surprisal explained more variance in Number of Homophones in the artificial lexica overall. 


\section{WHY DO HUMAN LANGUAGES HAVE HOMOPHONES?}

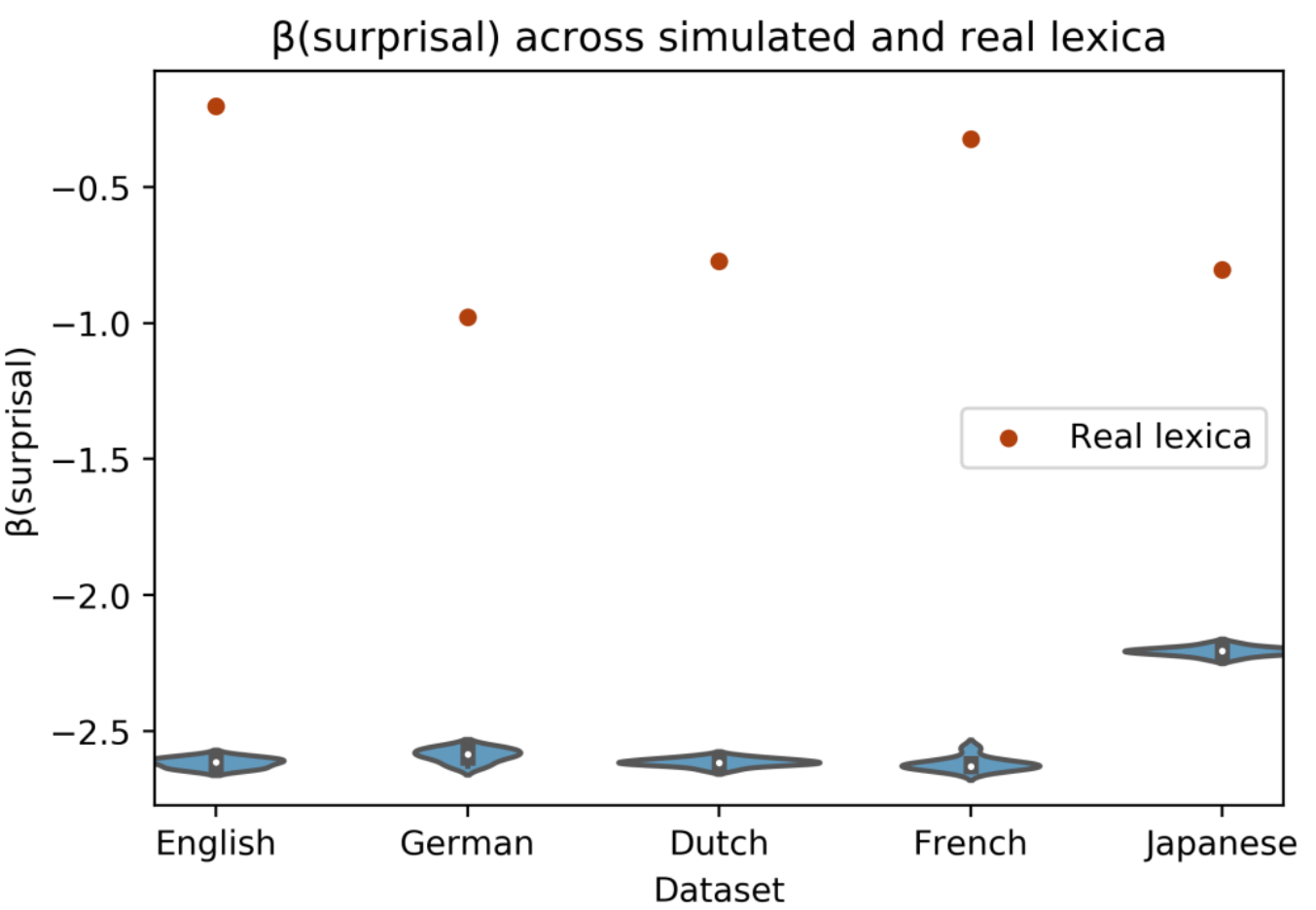

828

Dataset

829 Supplementary Figure 2: The coefficients for Surprisal were stronger (more negative) for all the artificial lexica. That is,

830 phonotactic plausibility was a better predictor of Number of Homophones in the artificial than real lexica.

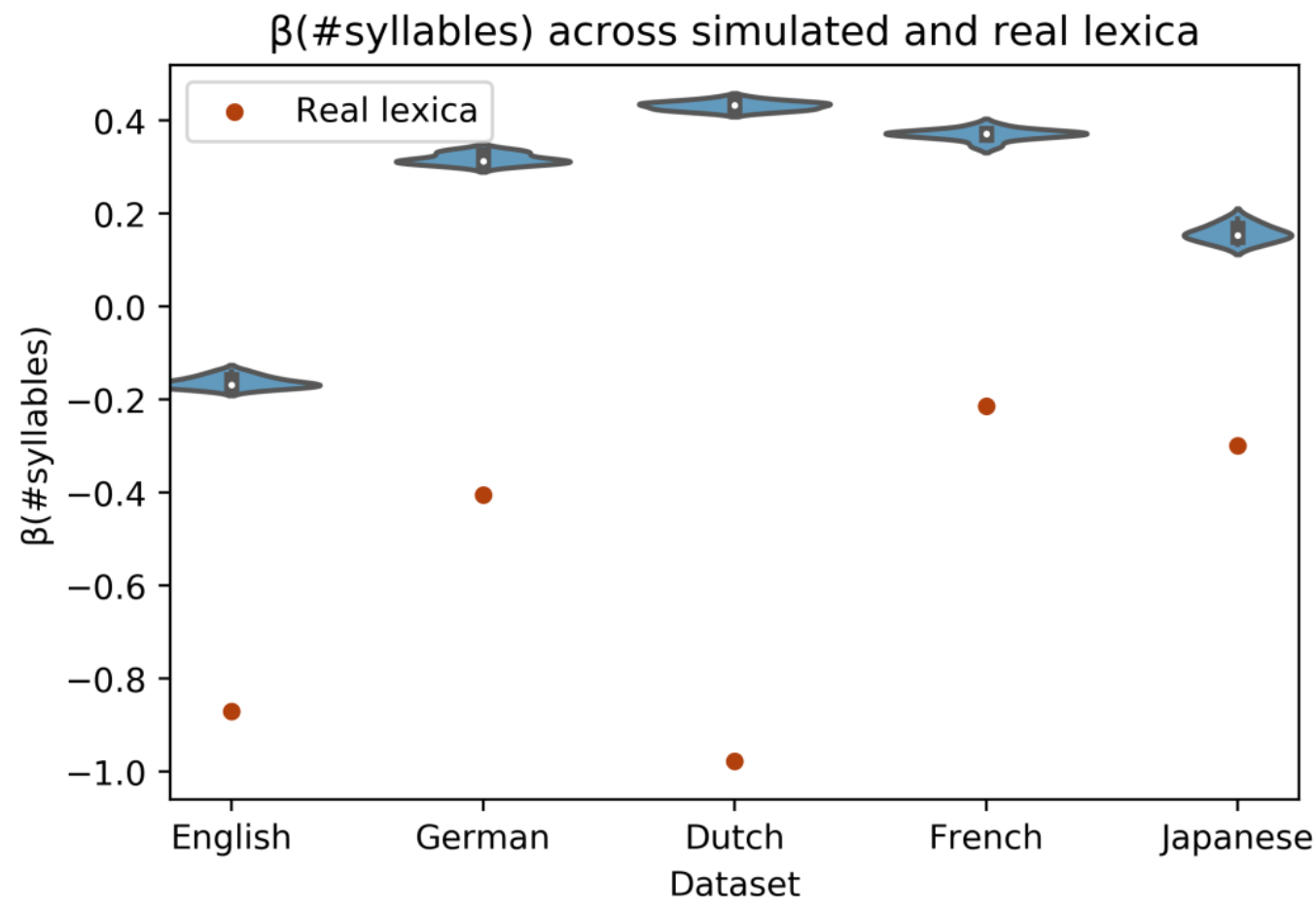

832 Supplementary Figure 3: The coefficients for Number of Syllables were more positive in the artificial lexica for the artificial 833 lexica, when included in a model with non-Normalized Surprisal. As explained in the main text, this is likely due to greater 834 collinearity between Surprisal and Number of Syllables for the artificial lexica. 
WHY DO HUMAN LANGUAGES HAVE HOMOPHONES?

835

836

837

838

839

840

841

842 


\section{WHY DO HUMAN LANGUAGES HAVE HOMOPHONES?}

\section{Supplementary Analysis 3: Calculating surprisal on held-out wordforms in the real lexica.}

844 The analyses performed on the real lexica replicated the negative relationship between Number

845 of Syllables and Number of Homophones reported by Piantadosi et al (2012), but failed to

846 replicate the relationship between Normalized Surprisal and Number of Homophones, which was

847 negative in Dutch and German, and insignificant in English; in all of the real lexica, we found

848 positive relationships between Normalized Surprisal and Number of Homophones. (In contrast,

849 all the artificial lexica showed negative relationships between Normalized Surprisal and Number

850 of Homophones.)

851 One explanation for this disparity in the results for the real lexica is that Piantadosi et al

852 (2012) calculated surprisal on held-out wordforms. That is, a phonotactic model was trained

853 using some larger set of wordforms, and was then used to calculate surprisal for wordforms that

854 did not appear in the training set. This approach has the advantage of correctly assigning very

855 low probabilities to rare wordforms. For example, if the wordform bnick appeared in the English

856 lexicon but no other words contained the onset $b n-$, it would receive lower probability (i.e.,

857 higher surprisal) if it was excluded from the set of wordforms used to train a phonotactic model.

858 Overall, in fact, surprisal estimates should be larger (i.e., lower probability) for wordforms

859 excluded from the training set — but particularly for wordforms with rarer phoneme sequences. In

860 principle, this could account for the disparity in results: if the probability of wordforms with rare

861 phoneme sequences is inflated, and if wordforms with rare phoneme sequences are less likely to

862 have homophones, then the relationship between Normalized Surprisal and Number of

863 Homophones could be weaker or even positive - assuming the probabilities of rare sequences are

864 inflated to such a degree as to be comparable with more frequent sequences that are also

865 associated with more homophones. 


\section{WHY DO HUMAN LANGUAGES HAVE HOMOPHONES?}

To address this concern, we recalculated Surprisal estimates for each real lexicon using

867

868

869

870

871

872

873

874

875

876

877

878 estimates for Normalized Surprisal for each of the real lexica (see Supplementary Figure 4), and

879 in some cases (e.g., Japanese), this coefficient was now negative $(\beta=-.37)$, as would be

880 predicted by a lexicon that recycles the most probable wordforms. However, the coefficient was

881 still positive in Dutch $(\beta=.38)$ and German $(\beta=.196)$, and close to 0 for English $(\beta=.001)$ and

882 French $(\beta=-.04)$.

883

884

885

886

887

This is in stark contrast to the coefficients for Normalized Surprisal for the artificial

lexica, which were all strongly negative (see Supplementary Figure 4). For example, the mean coefficient value across the artificial Dutch lexica was -5.01 $(\mathrm{SD}=.07)$. Similarly, although

$\beta \#$ syllables and pseudo- $\mathrm{R}^{2}$ values were slightly different across the real lexica with the new measure of held-out surprisal, each measure was reliably smaller in magnitude than the artificial lexica.

\footnotetext{
${ }^{11}$ 5-phone in the case of English, Dutch, and German; and 4-phone in the case of Japanese and French.
} 


\section{WHY DO HUMAN LANGUAGES HAVE HOMOPHONES?}

888 That is, the artificial lexica exhibited a stronger, more negative relationship between Number of

889 Syllables and Number of Homophones (Supplementary Figure 5) and better model fit overall, as

890 measured by pseudo-R² (Supplementary Figure 6).

891 There are two conclusions to draw from this supplementary analysis. First, the disparity

892 between our original results for Normalized Surprisal and those of Piantadosi et al (2012) could

893 be partially due to the fact that our initial measure was not calculated using a held-out set. Using

894 a held-out set, the estimates for Normalized Surprisal are closer to 0 for each language. (As noted

895 in the manuscript, other possible explanations include the use of a 5-phone or 4-phone model to

896 represent the language's phonotactics, and our use of types, rather than tokens, to train the

897 model.)

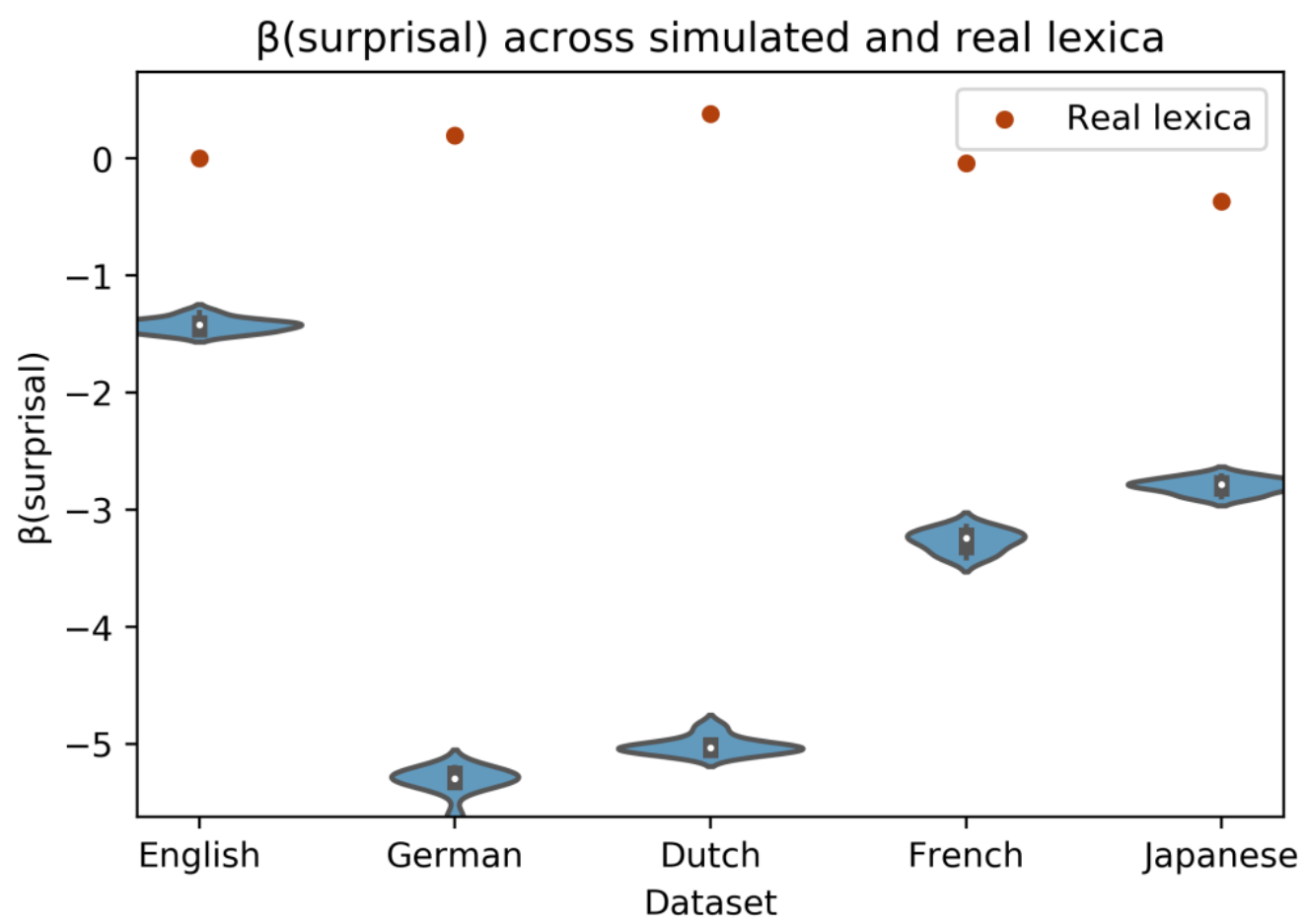

898

899 Supplementary Figure 4: The coefficients for Normalized Surprisal were more negative for the artificial lexica than the real

900 lexica, even when using an analogous held-out procedure on the real lexica. 


\section{WHY DO HUMAN LANGUAGES HAVE HOMOPHONES?}

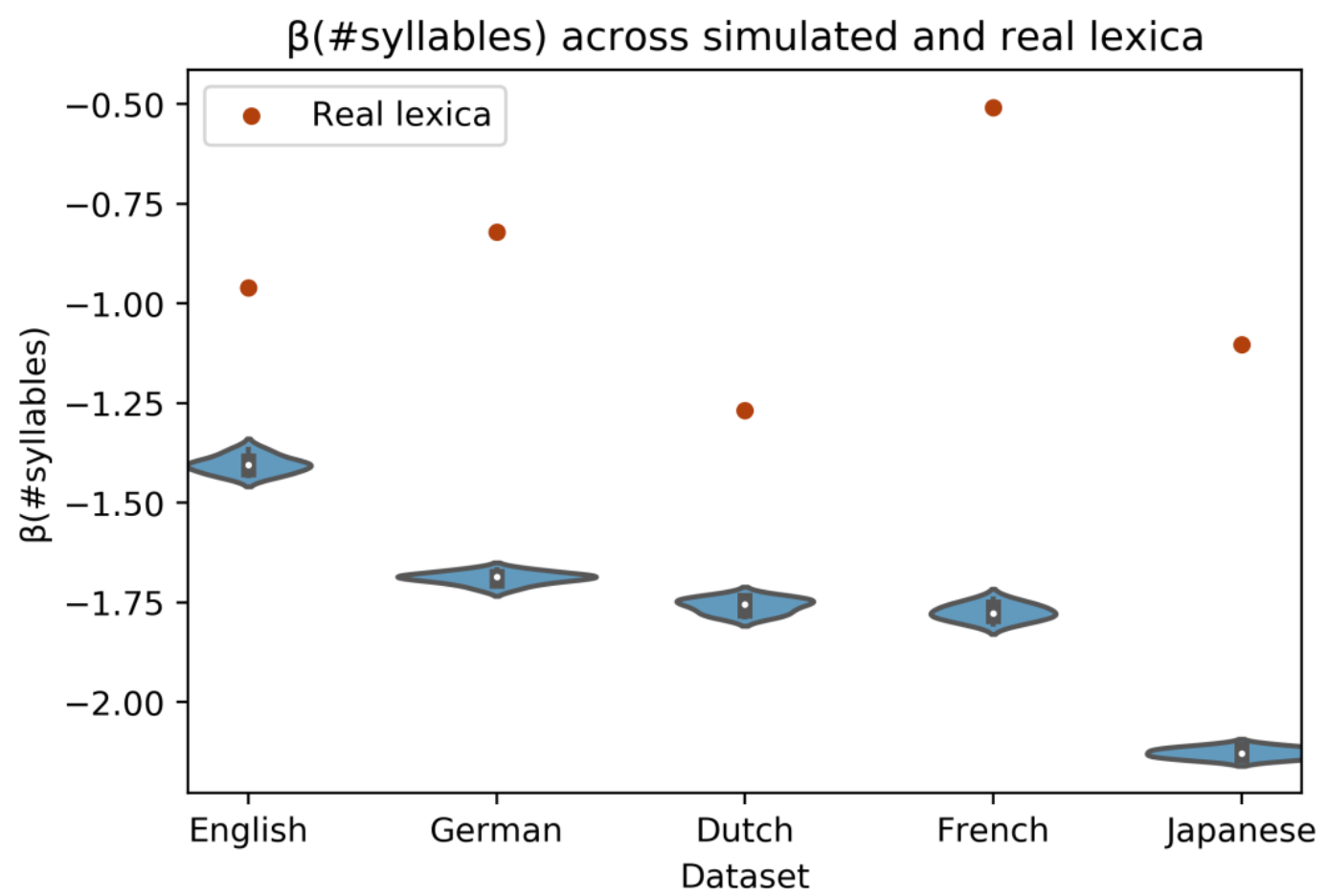

901

902 Supplementary Figure 5: Coefficients for Number of Syllables were stronger (more negative) for the artificial lexica than real 903 lexica.

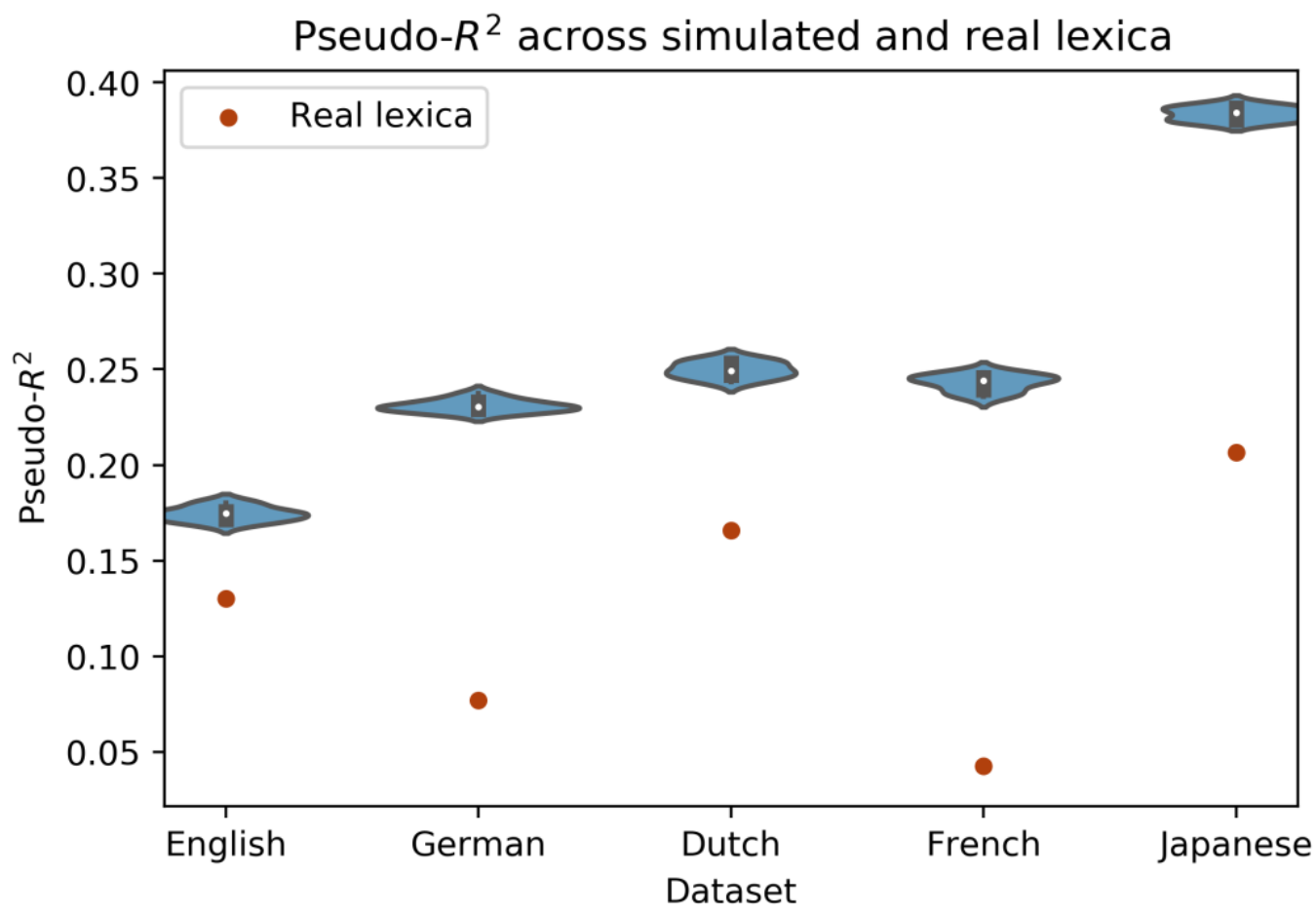

904

905 Supplementary Figure 6: Pseudo-R², a measure of model fit, was higher for all the artificial lexica across languages than their 906 real counterparts. 


\section{WHY DO HUMAN LANGUAGES HAVE HOMOPHONES?}

\section{Supplementary Analysis 4: Exploring the role of derivational morphology.}

909 One notable difference between the real and artificial lexica is that wordforms in the artificial

910 lexica were not constructed using processes of morphological composition. While the real lexica

911 contained only lemmas, some of those lemmas were morphologically complex, containing two or

912 more morphemes. In the primary manuscript, we suggested that derivational morphology could

913 be a mechanism by which real lexica indirectly avoid homophony - allowing them to reuse

914 lexical material without recycling the entire wordform. This would help explain why the real

915 lexica have fewer homophones than the artificial lexica.

916 However, as suggested by one anonymous reviewer, it is also possible that derivational

917 morphology is responsible for creating homophones. If this is true, and if morphologically

918 complex wordforms also tend to be longer, then derivational morphology could be responsible

919 for longer homophones in real lexica. This is one potential explanation for why the slope

920 coefficient for Number of Syllables is smaller in the real lexica than the artificial lexica-that is,

921 the real lexica have a higher proportion of long homophones overall, and have relatively less

922 short homophones, so the slope will be less steep than in the artificial lexica (which have more

923 short homophones and fewer long homophones). This would in turn suggest that real lexica do

924 not necessarily select against homophony outright, but redistribute homophony in particular 925 ways.

926 First, it is important to note that the real lexica do have fewer homophones overall, and

927 those homophones are more concentrated among short, phonotactically probable wordforms.

928 This means the discrepancy in slope coefficients cannot merely be due to the lack of derivational

929 morphology in artificial lexica. The fact that artificial lexica have more short homophones than 


\section{WHY DO HUMAN LANGUAGES HAVE HOMOPHONES?}

930 real lexica can thus be at least partially attributed to some form of selection pressure (whether

931 direct or indirect) against homophony in real lexica.

932 Here, we ask whether derivational morphology is indeed responsible for creating

933 homophones in real lexica, and whether those homophones tend to be longer rather than shorter.

934 This account makes several testable predictions; we consider the evidence for each in turn,

935 focusing on English and French (for which we have information about morphological complexity 936 readily available).

937 First, in real lexica, the set of multimorphemic wordforms could have more homophones

938 (or a higher proportion of homophones) overall. However, at least in English and French,

939 monorphemic wordforms actually have a higher Mean Number of Homophones per wordform,

940 by a factor of about 2x (in French: 0.2 vs. 0.1) or 9x (in English: 0.38 vs. 0.04).

941 Of course, it may not be that multimorphemic wordforms have more homophones

942 overall, but rather, derivational morphology is most likely to generate homophones among long

943 wordforms. If true, this account predicts that removing multimorphemic wordforms from the real

944 lexicon should result in a reduction in the Mean Number of Homophones per wordform among

945 long wordforms in particular. This could be measured by an increase in the slope coefficient for

946 Number of Syllables when considering only monomorphemic wordforms (i.e., excluding

947 multimorphemic wordforms). That is, if derivational morphology is most likely to create longer

948 homophones, then excluding multimorphemic wordforms from the lexicon should

949 disproportionately remove homophones among long wordforms — resulting in a lexicon with

950 relatively more short homophones, and thus a steeper slope between Number of Syllables and

951 Number of Homophones. 


\section{WHY DO HUMAN LANGUAGES HAVE HOMOPHONES?}

In fact, the opposite is true in both English and French. The relationship between Number

953 of Syllables and Number of Homophones is weaker (less negative) when multimorphemic

954 wordforms are removed, i.e., only monomorphemic wordforms are used in the analysis. In

955 English, the slope coefficient for Number of Syllables is weaker when considering

956 monomorphemic wordforms only $[\beta=-0.62, \mathrm{SE}=.02]$ than when using the full lexicon $[\beta=-$

$9570.96, \mathrm{SE}=.02]$. Similarly, in French, the slope coefficient for Number of Syllables is smaller

958 when including only monomorphemic wordforms $[\beta=-0.42, \mathrm{SE}=.02]$ than when using the full

959 lexicon $[\beta=-0.49, \mathrm{SE}=.01]$.

960 In other words, it appears that the presence of derivational morphology is correlated with

961 both a decrease in the Mean Number of Homophones per wordform, and in a relative reduction

962 in the concentration of homophony among long wordforms in particular. Thus, the evidence does

963 not support the claim that derivational morphology explains why the real lexica have fewer short

964 homophones than the artificial lexica; if anything, it is more consistent with the argument that

965 derivational morphology serves as a mechanism by which outright homophony can be avoided in

966 the lexicon. 


\section{WHY DO HUMAN LANGUAGES HAVE HOMOPHONES?}

\section{Supplementary Analysis 5: Generating lexica with a 50/50 split}

969 In the primary manuscript, artificial lexica were generated by first training an $n$-phone

970 phonotactic model on the complete set of unique wordforms in the target language, then using

971 this model to generate a series of artificial lexica matched for the distribution of word lengths in

972 the real lexicon. One concern with this approach is that the same phonotactic model was used to

973 both generate and evaluate wordforms in each artificial lexicon. This could have two effects.

974 First, estimates of Surprisal will likely be lower overall (see also Supplementary Analysis 3).

975 Second, estimates of the relationship between Surprisal and Number of Homophones could be

976 systematically biased, possibly in the direction of inflating this relationship.

977 To address this concern, we followed the advice of an anonymous reviewer to generate

978 and evaluate the artificial lexica using a hold-out procedure. For each language, the real lexicon

979 was randomly divided into two halves, which in turn were used to train two separate phonotactic

980 models. $50 \%$ of wordforms in the real lexicon were used to train a generative model (i.e., to

981 generate wordforms for each corresponding artificial lexicon), and 50\% were used to train an

982 evaluative model (i.e., to evaluate the phonotactic probability newly created wordforms). This

983 means that the models used to estimate Surprisal were trained using an orthogonal set of

984 wordforms. Of course, each $50 \%$ of the real lexicon should follow the same phonotactic rules,

985 but this eliminates the concern of overfitting to the same wordforms for both generation and

986 evaluation. All other aspects of the generation process were identical: 10 artificial lexica were

987 generated for each language, matched for the distribution of word lengths in the original lexicon.

988 As in the primary manuscript, we compared each real lexicon to the 10 artificial lexica along

989 multiple dimensions: three descriptive measures (Maximum Number of Homophones, Mean

990 Number of Homophones, and Homophony Rate), and three measures derived from a Poisson 


\section{WHY DO HUMAN LANGUAGES HAVE HOMOPHONES?}

991 model regressing Number of Homophones against Normalized Surprisal and Number of

992 Syllables.

$993 \quad$ Results.

994 As in the original analyses, the artificial lexica all exhibited upper-bounds on the

995 Maximum Number of Homophones per wordform, as well as a larger number of Mean

996 Homophones per wordform. The Homophony Rate was also higher in the artificial lexica for

997 English, German, and Dutch, but as in the original analyses, not for French and Japanese (see

998 Supplementary Table 1 below for descriptive statistics for each real and artificial lexica).

\begin{tabular}{|c|c|c|c|c|c|}
\hline & English & German & Dutch & French & Japanese \\
\hline \multirow{2}{\text{Maximum}}{$\begin{array}{c}\text { Number of } \\
\text { Homophones }\end{array}$} & 7 & 4 & 5 & 12 & 33 \\
\cline { 2 - 6 } & $27(3.77)$ & $48.3(8.82)$ & $94.2(10.01)$ & $53.1(7.67)$ & $78.5(6.996)$ \\
\hline $\begin{array}{c}\text { Mean Number } \\
\text { of } \\
\text { Homophones }\end{array}$ & $0.29(0.002)$ & $0.18(0.001)$ & $0.28(0.003)$ & $0.22(0.003)$ & $0.28(0.002)$ \\
\cline { 2 - 6 } & 0.16 & 0.02 & 0.03 & 0.14 & 0.15 \\
\cline { 2 - 6 } $\begin{array}{c}\text { Homophony } \\
\text { Rate }\end{array}$ & $0.17(0.001)$ & $0.1(0.001)$ & $0.12(0.001)$ & $0.11(0.001)$ & $0.11(0.001)$ \\
\hline
\end{tabular}

999

1000 Qualitatively similar results were also obtained when comparing the coefficients and

1001 pseudo- $\mathrm{R}^{2}$ values across the real and artificial lexica. Once again, coefficients for Number of

1002 Syllables were larger (more negative) for the artificial lexica than in the real lexica (see

1003 Supplementary Figure 7 below), indicating that homophony was even more concentrated among

1004 short wordforms in the artificial lexica. 


\section{WHY DO HUMAN LANGUAGES HAVE HOMOPHONES?}

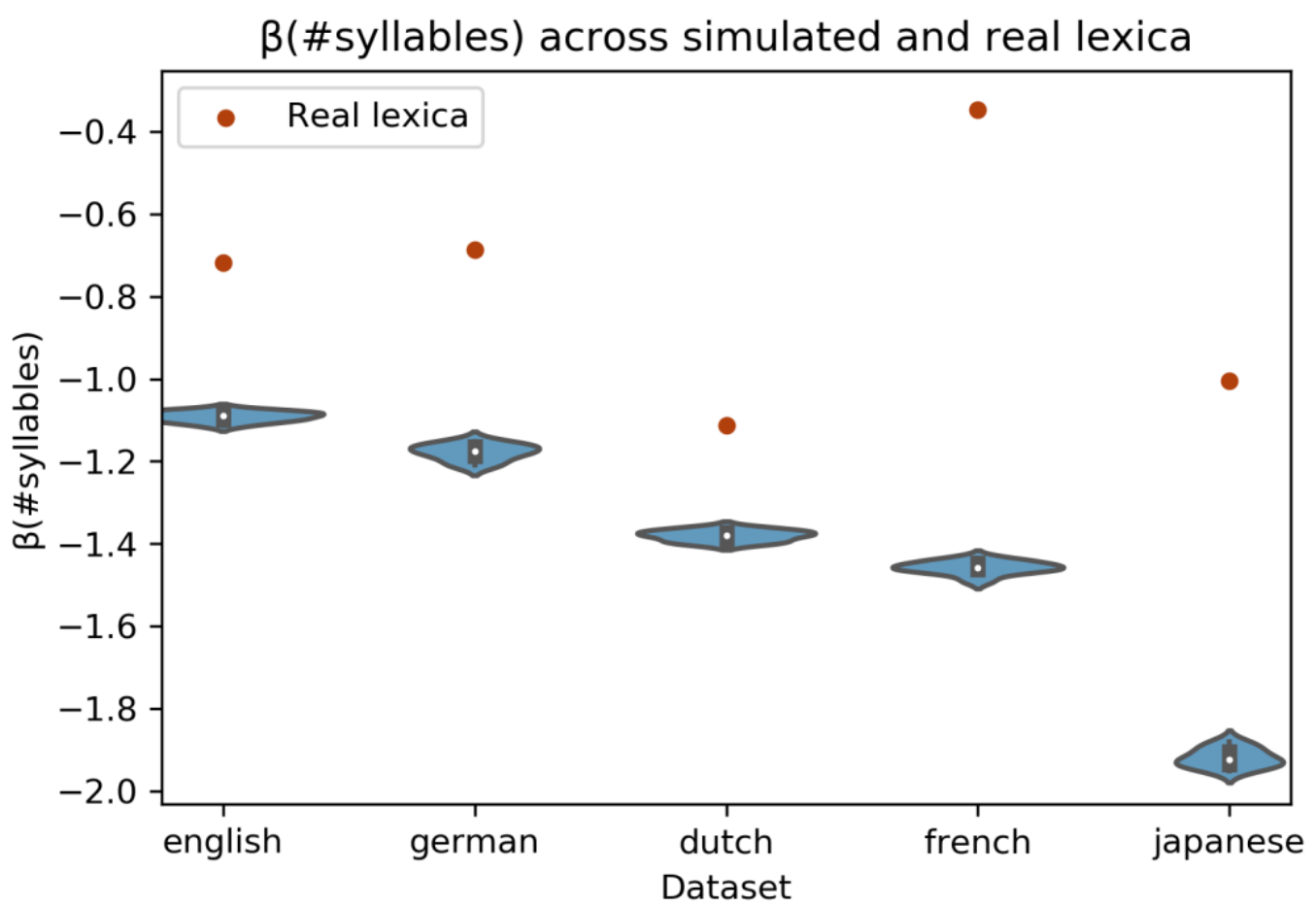

1005

Supplementary Figure 7: Number of Syllables was more strongly (negatively) related to Number of Homophones across all the

\section{7 artificial lexica than the real lexica.}

1008

1009 Similarly, pseudo- $\mathrm{R}^{2}$ values were higher across all the artificial lexica (see Supplementary

1010 Figure 8 below), indicating that both predictors combined (Normalized Surprisal and Number of

1011 Syllables) produced better model fit in the simulated lexica than the real lexica. 


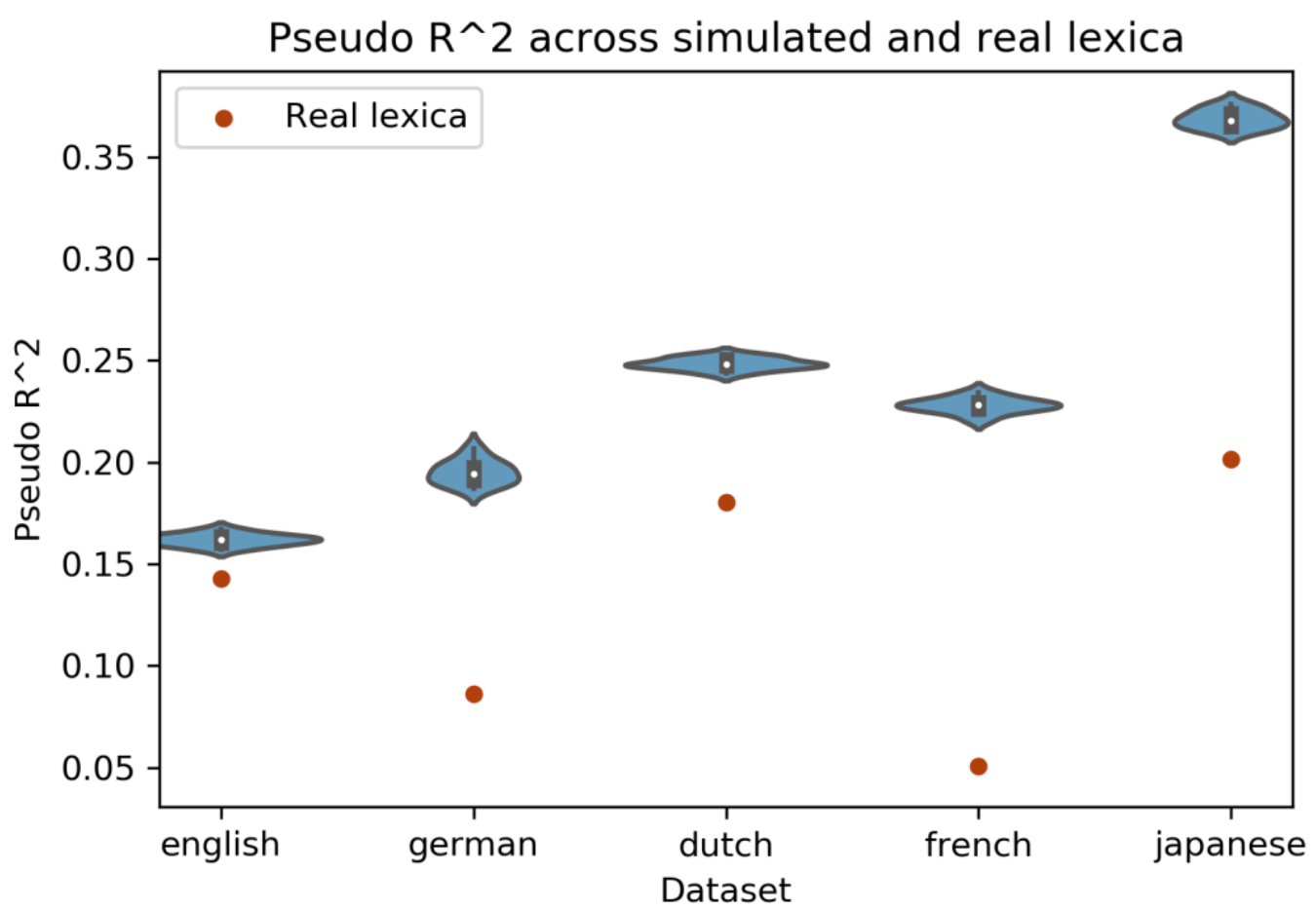

1012 Dataset

1013 Supplementary Figure 8: Values for pseudo- $R^{2}$, a measure of model fit, were larger in each of the artificial than real lexica, 1014 suggesting that Number of Syllables and Normalized Surprisal combined were better predictors of homophony in the artificial 1015 baselines.

1016 Finally, and most importantly, the coefficients for Normalized Surprisal were larger in

1017 the expected direction (negative), for each of the artificial lexica than the corresponding real

1018 lexicon. For example, the coefficient for Normalized Surprisal in the real English lexicon was

1019 positive $[\beta=0.78]$, while the artificial lexica all exhibited negative relationships $[\mathrm{M}=-0.48, \mathrm{SD}$

$1020=0.02]$. Similarly, the real Japanese lexicon exhibited a very weak relationship between

1021 Normalized Surprisal and Number of Homophones $[\beta=0.0004]$, while this relationship was

1022 strongly negative in each artificial lexica $[\beta=-1.65, \mathrm{SD}=0.06]$. (See Supplementary Figure 9

1023 for an illustration of this effect across languages). 


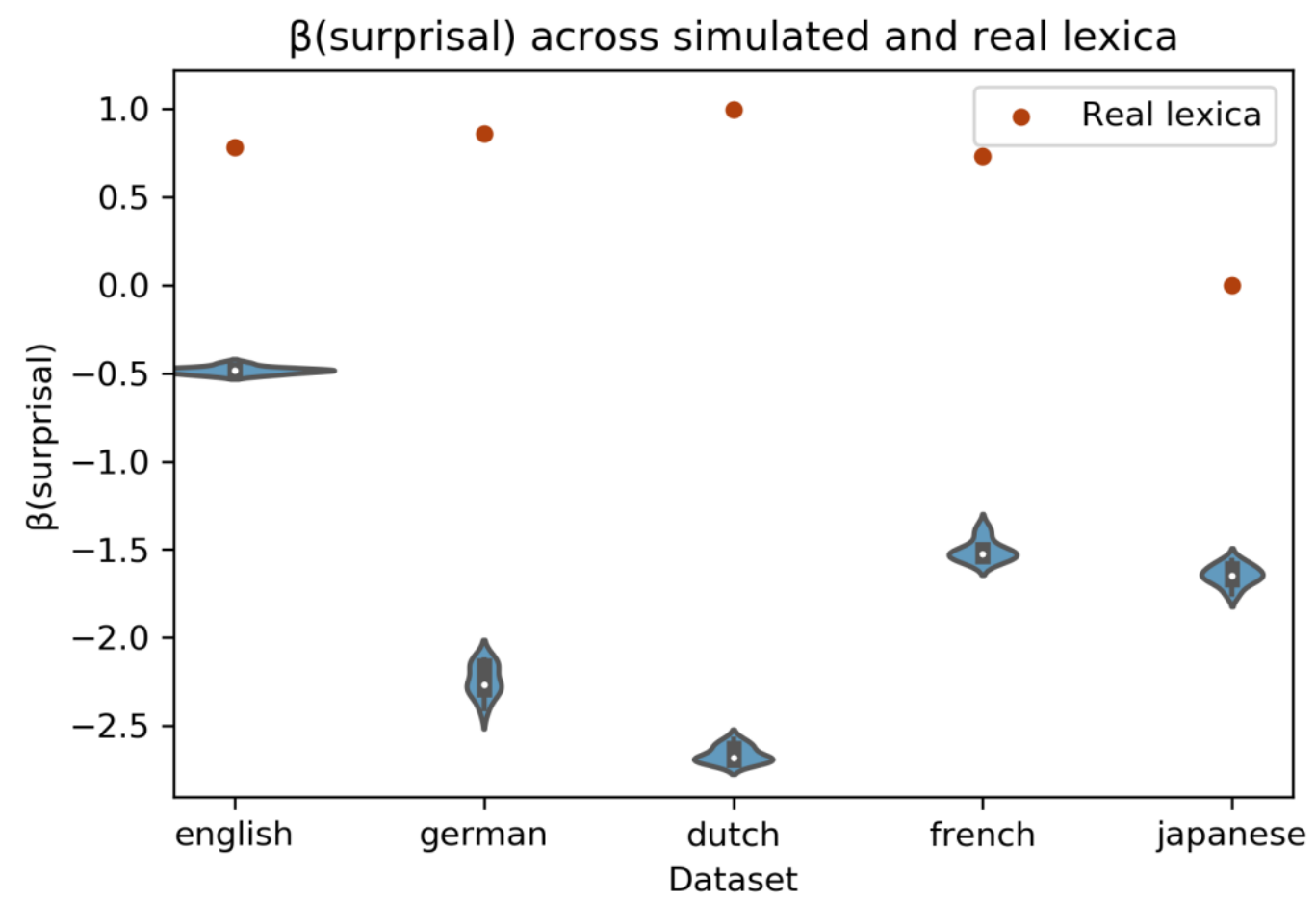

1024

Supplementary Figure 9: Normalized Surprisal exhibited more negative relationships in each of the artificial lexica than the real lexica. That is, homophony was more concentrated among phonotactically probable wordforms in the artificial baselines than in the real languages.

1028 Also importantly, this qualitative effect holds regardless of whether Normalized Surprisal

1029 is calculated using a hold-out set for the real lexica as well. As shown in Supplementary Analysis

10303 , using a measure of held-out surprisal did result in smaller (closer to 0) coefficient estimates

1031 for Normalized Surprisal for each real lexica (see Supplementary Figure 4). In each case,

1032 however, these coefficient estimates were still smaller (less negative) than the coefficients in the

1033 corresponding artificial lexica - whether or not those lexica were generated using the 50/50 split

1034 described here.

1035 